\title{
Water, Nutrient, and Energy-use Efficiencies of No-till Rainfed Cropping Systems with or without Residue Retention in a Semi-Arid Dryland Area
}

\author{
Lal P. Amgain ${ }^{a, *}$, Ajit R. Sharma ${ }^{b}$, Jagadish Timsina ${ }^{c}$, and Pradeep Wagle ${ }^{d}$ \\ ${ }^{a}$ Faculty of Agriculture Science, Far Western University, Tikapur, Kailali, Nepal \\ ${ }^{b} R L B$ Central Agricultural University, Jhansi - Gwalior Road, Jhansi 284002 UP, India \\ ${ }^{c}$ Institute for Studies and Development Worldwide, Homebush West, Sydney, NSW 2140, Australia \\ ${ }^{d}$ USDA, Agricultural Research Service, Grazinglands Research Laboratory, El Reno, OK 73036, USA
}

\section{MANUSCRIPT INFO}

Article history:

Received 9 Apr 2019

Received in revised form 10 Nov 2019

Accepted 12 Nov 2019

Keywords:

Apparent nutrient balance

Consumptive water use

Energy inputs and outputs

Energy-use efficiency

Water-use efficiency

\begin{abstract}
A B S T R A C T
No-till rainfed cropping systems are being considered by farmers to make farming more profitable by reducing production costs, thereby enhancing resource-use efficiency. Field studies were conducted at the Indian Agricultural Research Institute (IARI), New Delhi during rainy and winter seasons of 2010-2011 and 2011-2012 to examine consumptive use of water (CW), water-use efficiency (WUE), nutrient uptake and balance, and energy-use efficiency (EUE) of nine diverse cropping systems based on three rainy season crops - pearl millet (Pennisetum glaucum (L.) R. Br.), cluster bean (Cyamopsis tetragonoloba L.), and green gram (Vigna radiata L. Wilczek) followed by three winter crops - wheat (Triticum aestivum L.), chickpea (Cicer arietinum L.), and mustard (Brassica juncea L.) in each of those three rainy season crop planted fields under no-till semi-arid rainfed conditions. Three residue treatments [i.e., no residue, crop residue, and Ipil-ipil \{Leucaena leucocephala (Lam) twigs\}] were examined for both rainy season and winter crops. Retention of crop residues significantly increased soil moisture, CW, and WUE in all cropping systems. Good growth of mustard, chickpea, and wheat after cluster bean, and a large amount of cluster bean green pods resulted in substantially higher $\mathrm{CW}$ and WUE of cluster bean-based systems compared to pearl milletand green gram-based systems. Crop nutrient uptake increased substantially under crop residue and Leucaena twigs treatments compared to no-residue control plots due to enhanced crop growth and augmentation of nutrients. However, nutrient uptake and apparent nutrient balances varied greatly among cropping systems. Energy input requirement increased by approximately 10 times under crop residue and Leucaena twigs treatments. As a result, net energy balance and EUE were substantially higher for no-residue treatments. Leucaena twigs treatments had higher net energy balance and EUE than crop residue treatments, indicating the importance of leguminous residues in crop production. Results indicate the necessity of exercising optimal balance between retention of crop residues and energy inputs for the long-term soil health and sustainability of cropping systems.
\end{abstract}

(c) 2019 NAPA. All rights reserved.

\section{Citation:}

Amgain, L. P., Sharma, A. R., Timsina, J., \& Wagle, P. (2019). Water, nutrient, and energy-use efficiencies of no-till rainfed cropping systems with or without residue retention in a semi-arid dryland area. Global Journal of Agricultural and Allied Sciences, 1(1), 30-42.

\section{Introduction}

Due to declining water availability for agriculture, rainfed agriculture is gaining importance worldwide as it covers about $80 \%$ of the global agricultural area and shares about $60 \%$ of the global food-grain production (Rockström et al., 2010). The future prosperity of India relies on rainfed agriculture as $67 \%$ of $143 \mathrm{M}$ ha net cultivated area, $91 \%$ of coarse grains and pulses, $80 \%$ of oilseeds, $60 \%$ of cotton (Gossypium hirsutum L.), $50 \%$ of rice (Oryza sativa L.), and 19\% of wheat (Triticum aestivum L.) grown areas are under rainfed conditions, most dominantly in the semi-arid drylands of north-western India (Gupta, Jat, Gopal \& Kumar, 2010; Prasad \& Bhatia, 2009).

Conventional agriculture with intensive conventional tillage (CT) systems with the use of heavy machinery can lead to a decrease in soil organic matter (SOM), loss of soil structure and fertility, and overall deterioration of soil health (Pingali, Vignozzi \& Pellegrini, 2004). In comparison, conservation agriculture (CA) systems involve minimum soil disturbance, maintain soil cover through crop residues or other mulching materials, and follow dynamic crop rotations for achieving higher

*Corresponding author. E-mail address: lalamgain@fwuagriculture.com 
productivity and sustainability (Hobbs, 2007; Sayre \& Hobbs, 2004). The CA systems have gained importance globally as they are more energy efficient and beneficial to the environment as compared to conventional systems (Filipovic, Silvio, Zlatko, Robert \& Djuro, 2006; Hariram, Saimi, Kler, Timsina \& Humphreys, 2012; Sharma, Chokkar, Rani, Gathis \& Kumar, 2002). The CA systems, including no-till (NT) practices, save fuel energy, restrict release of soil organic carbon (SOC), and mitigate carbon dioxide concentration in the atmosphere (Grace, Jain, Harrington \& Philip, 2003; Rao, Singh, Joshi \& Ramakrishna, 2000; Saha et al., 2010). Thus, NT practices have a great potential to sequester carbon, increase SOM, minimize soil erosion, and reduce production costs by maintaining a similar production level (Gathala et al., 2011a, 2016; Rao et al., 2000). Research findings from several locations in the Indo-Gangetic plains of South Asia showed saving of land preparation costs by about US\$25-50 ha- and reductions in diesel consumption by 50-60 liters ha ${ }^{-1}$ with NT compared to CT (Kumar et al., 2013a, 2013b; Sangar, Abrol \& Gupta, 2005). Furthermore, wheat yields increased up to $30 \%$ by using NT with optimal level of energy input (Chaudhary, Gangwar \& Pandey, 2006, Saharawat et al., 2010).

Retention of crop residues on the soil surface creates a physical barrier to the emergence of weeds, moderates soil temperature fluctuations, conserves soil moisture, adds SOM, improves nutrient-water interactions, and reduces air pollution arising due to large-scale burning of crop residues (Bhusan \& Sharma, 2002; Sharma \& Acharya, 2000; Sharma et al., 1995). When the residues are retained on the soil surface in combination with NT practices, the enhanced biological processes lead to improved soil quality (Reicosky, 2003). The importance of using the pruned materials of various trees and shrubs grown in non-cropped alley lands as brought-in residues or mulch since pre-historic time as in the Vedas and Kuran is reflected from several records, and these practices are still dominant in highlands and rainfed areas in India (Dhyani, Newaj \& Sharma, 2009).

The CA system is now adopted globally on about $120 \mathrm{M}$ ha, largely in rainfed areas (Derpsch \& Friedrich, 2009). However, only about $2 \mathrm{M}$ ha of wheat is cultivated with NT seed drills in India (Aryal, Sapkota, Jat \& Bishnoi, 2015; Jat et al., 2014). In irrigated areas of north-western India, about 20 diversified cropping systems are practiced (Gill \& Ahlawat, 2006), but few cropping systems with their inconsistent performance in terms of productivity, profitability, and energetics have been documented for rainfed areas. Of the few studies conducted with rainfed cropping systems, the cluster bean (Cyamopsis tetragonoloba L.)-mustard (Brassica juncea L.) system was more remunerative than the cluster bean-wheat system in Hisar, north-west India, while the cluster bean-wheat system had higher net returns with higher water-use efficiency (WUE, the efficiency to gain carbon per unit of water) in Gwalior, central India (Saxena, Singh \& Joshi, 1997; Singh, Sharma, Deo, Siag \& Verma, 1998). Likewise, net returns and the benefit-cost ratio were higher with the green gram (Vigna radiata $\mathrm{L}$. Wilczek)-wheat system in Rajasthan, northern India (Singh, Singh \& Patidar, 2008). Similarly, on-farm experiments conducted under rainfed conditions at 35 locations in five districts of Rajasthan revealed that the cluster bean-wheat sequence yielded the highest gross returns, followed by the cluster bean-mustard and pearl millet (Pennisetum glaucum (L.) R. Br.)-wheat crop sequences (Lal, Bhati \& Nag, 2004). There are also records of remarkable increases in crop yields in the maize (Zea mays L.)-wheat system with scanty rainfall through the maintenance of appropriate vegetative cover in rainfed areas (Acharya, Kapur \& Dixit, 1998; Sharma \& Acharya 2000; Sharma, Singh, Tyagi \& Mohan, 1998; Sharma et al., 2010). Incorporation of Ipil-ipil (Leucaena leucocephala (Lam)) twigs was effective for both rainy season and winter crops due to their high nitrogen
(N) content and availability (Sharma \& Behera, 2009; Sharma et al., 2010, Sharma, Singh, Dhyani \& Dube, 2011), with significant residual effects on increasing the soil fertility and productivity of subsequent crops (Jones, Wendt, Bunderson \& Itimu, 1996; Lehria, Bali \& Singh, 2006). Despite several instances of sustainable productivity and profitability of rainfed cropping systems following the CA, its adoption under rainfed conditions has been slow (Pittelkow et al., 2014).

As water is the scarce and costly input for crop production in semi-arid rainfed areas, it is important to increase crop productivity and WUE of rainfed cropping systems. A distinct advantage of NT systems is that they generally maintain or increase soil macro pores and SOM content, thereby increasing the water-holding capacity of soil (McMaster, Palic \& Dunn, 2002). The annual rainfall of $600-800 \mathrm{~mm}$ in most of the semi-arid rainfed areas may be adequate for crop growth, but its uneven distribution results in deficit moisture stress and low yields in dry rainfed areas. Thus, the development of more innovative region-specific CA systems could be an alternative approach for boosting the productivity and increasing the resource (i.e., water, fertilizer, energy) use efficiencies (Gathala et al., 2011a, 2013) for those areas. In addition, the complexities associated with residue management in NT systems indicate the need for more research for efficient utilization of crop residues. Therefore, this study was undertaken to quantify the influence of nine diverse NT rainfed double cropping systems (three rainy season crops - pearl millet, cluster bean, and green gram followed by three winter crops - wheat, chickpea (Cicer arietinum L.), and mustard under three residue treatments (i.e., no residue, crop residue, and Leucaena twigs) on water and nutrient uptake and balances, energy relations, and resource-use efficiencies in a semi-arid environment of north-west India.

\section{Materials and Methods}

\subsection{Study Site, Soil, and Weather Details}

Field experiments were conducted in a one-hectare field located at the Research Farm of the Indian Agricultural Research Institute (IARI), New Delhi $\left(28.4^{\circ} \mathrm{N}, 77.1^{\circ} \mathrm{E}, 229\right.$ masl) during rainy (June-October) and winter (October-March) seasons of 2010-2011 and 2011-2012. The soil type was shallow $(\sim 15 \mathrm{~cm})$ in depth with sandy-loam texture, bulk density of 1.55 $\mathrm{Mg} \mathrm{m}^{-3}$, and field capacity of $18.68 \%(\mathrm{w} / \mathrm{w})$. It had $0.40 \%$ organic carbon (C), $147.2 \mathrm{~kg} \mathrm{ha}^{-1} \mathrm{KMnO}_{4}$-oxidizable $\mathrm{N}, 17.0 \mathrm{~kg} \mathrm{ha}^{-1} 0.5 \mathrm{~N} \mathrm{NaHCO}_{3}$ extractable phosphorus (P), $225.1 \mathrm{~kg} \mathrm{ha}^{-1} 1.0 \mathrm{~N} \mathrm{NH}_{4} \mathrm{OAc}$-exchangeable potassium $(\mathrm{K})$, and $7.5 \mathrm{pH}$ at the beginning of the experiment. The average annual rainfall of Delhi over a decade (2000-2010) was $739 \mathrm{~mm}$, of which $>80 \%$ occurred generally during the monsoon (rainy) period (JulySeptember). There was $\sim 44 \%$ higher rainfall in 2010-2011 (954 mm, 29\% higher than the mean for the past decade) than in 2011-2012 (662 mm, $\sim 10 \%$ lower than the mean for the past decade). Winter season of 20102011 received about $85 \mathrm{~mm}$ well-distributed rainfall, but there was only 34 $\mathrm{mm}$ sparsely distributed rainfall in winter season of 2011-2012. The average winter season rainfall for the past decade was $\sim 125 \mathrm{~mm}$. Overall, the study site experienced contrasting weather conditions during the two years of the study period.

\subsection{Management Practices and Treatment Details}

The experimental land was laser-leveled during November 2009 and a uniformity trial was conducted by growing wheat cv. 'PBW-175' to standardize the field prior to the beginning of the experiment. Thereafter, 
continuous NT was practiced to sow all six tested crops under rainfed conditions during the entire study period of 2010-2011 and 2011-2012. Pearl millet, cluster bean, and green gram were grown during the 2010 rainy season under no-residue, crop residues, and Leucaena twigs in a randomized complete block design (RCBD) with four replications. On each rainy season crop (pearl millet, cluster bean, and green gram) planted fields, three winter crops (wheat, chickpea, and mustard) were grown in strips during the winter season of 2010-2011. The resulting experimental design to study nine diverse rainfed cropping systems based on three rainy season crops (pearl millet, cluster bean, and green gram) followed by three winter crops (wheat, chickpea, and mustard) was a strip-split plot design (Figure 1). Pearl millet, wheat, and mustard were grown with 60:40:20 kg NPK ha1, while cluster bean, green gram, and chickpea were grown with 20:40:20 $\mathrm{kg}$ NPK ha ${ }^{-1}$. Diammonium phosphate (DAP, 18\% N and 46\% P) was applied in rows with Happy Seeder (Sidhu et al., 2007), while potassium chloride $(\mathrm{KCl}, 60 \% \mathrm{~K})$, and urea $(46 \% \mathrm{~N})$ were broadcasted before sowing. Pearl millet, wheat, and mustard were top-dressed with $50 \% \mathrm{~N}$ between 30 and 60 days after seeding (DAS) coinciding with rainfall. After harvesting, residues were left in the fields under crop residue treatment, but they were removed from no-residue and Leucaena twigs treatments. Crop residues were applied at the rate of $\sim 5.0 \mathrm{t}$ ha- ${ }^{1}$ dry matter and Leucaena, brought from nearby locations, were applied at the rate of $\sim 10.0 \mathrm{t} \mathrm{ha}^{-1}$ green twigs $\left(\sim 3.5 \mathrm{t} \mathrm{ha}^{-1}\right.$ dry matter, $65-70 \%$ moisture) seasonally for both rainy season and winter crops.

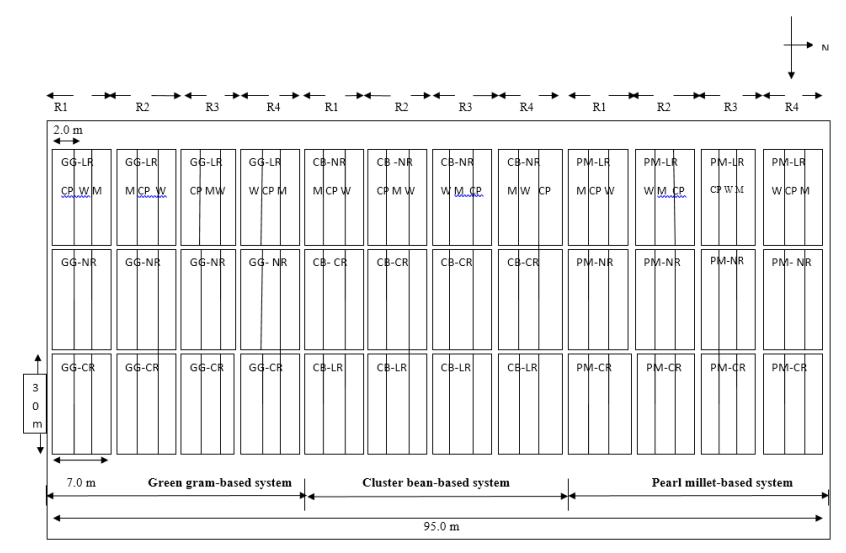

Figure 1. Layout of the experimental plot.

Note: $\mathrm{PM}=$ Pearl millet, $\mathrm{NR}=$ No residue, $\mathrm{CR}=$ Crop residue, $\mathrm{LT}=$ Leucaena twigs, $\mathrm{W}=$ Wheat, $\mathrm{CP}=$ Chickpea, and $\mathrm{M}=$ Mustard.

The seed and stover yields of rainy season crops were recorded from a $25 \mathrm{~m}^{2}$ area in 2010 and a $10 \mathrm{~m}^{2}$ area in subsequent seasons. Thinning was done in mustard to maintain plant-to-plant spacing of $8-10 \mathrm{~cm}$. In the second year (2011-2012), mustard and chickpea sown on October 3 did not germinate due to low soil moisture coinciding with the high daily maximum temperature and evaporation throughout October. Therefore, limited irrigation (about 200,000 liters or $200 \mathrm{~m}^{3}$ of water ha ${ }^{-1}$ ) through $2.5 \mathrm{~cm}$ diameter pipe from a nearby drain was applied on the seed-rows after 30 DAS to obtain uniform plant stands. Further, the gravimetric soil profile moisture in the surface soil $(0-15 \mathrm{~cm})$ was only $4-5 \%$ in the plots to be sown wheat at the end of October 2011. Therefore, a pre-sowing flood irrigation equivalent to $7.0 \mathrm{~cm}$ was given to these fields, and wheat was sown on November 11, 2011 after attainment of optimum moisture for planting. Under irrigated conditions, the biological $\mathrm{N}_{2}$ fixation potential of cluster bean, green gram, and chickpea have been reported to be 196, 50, and $46 \mathrm{~kg} \mathrm{ha}^{-1} \mathrm{yr}^{-1}$, respectively (Peoples, Herridge \& Ladha, 1995). In our study, only half of these values were added to determine total $\mathrm{N}$ inputs due to no inoculation with Rhizobium strains and moisture-deficient rainfed semi-arid conditions as in a previous study (Bandalucco et al., 2010).

\subsection{Consumptive Water Use and Water-use Efficiency}

Soil profile moisture at 0-15, 15-30, and 30-45 cm depths was measured at various growth stages of crops, from before sowing to after harvesting, using the gravimetric method. The effective rainfall was then added to the soil moisture to estimate the consumptive use of water (CW) (Allen, Pereira, Raes \& Smith, 1998). Soil moisture content at different depths (volume/volume) was calculated by multiplying with respective bulk density values. Water requirement of crops was calculated based on the soil moisture depletion, effective rainfall, and irrigation amount applied to the winter crops in the second year (2011-2012). Therefore, CW was estimated for each treatment using the following equation (Michael, 2014):

$C W=N I R+R_{f}+\sum \frac{n\left(M b_{i}-M e_{i}\right)}{100} \times A s_{i} \times D_{i}$

where $\mathrm{CW}=$ seasonal consumptive use of water $(\mathrm{mm}) ; \mathrm{NIR}=$ total irrigation water applied during the crop season $(\mathrm{mm}) ; \mathrm{R}_{\mathrm{f}}=$ seasonal rainfall $(\mathrm{mm}) ; \mathrm{Mb}_{\mathrm{i}}=$ percent moisture content at the beginning of the season of the $\mathrm{i}^{\text {th }}$ layer of the soil; $\mathrm{Me}_{\mathrm{i}}=$ percent moisture content at the end of the season of the $\mathrm{i}^{\text {th }}$ layer of the soil; $\mathrm{n}=$ number of soil layers considered within the root zone depth (this was considered for 0-15, 15-30, and 30-45 cm); $D_{i}=$ depth of the $\mathrm{i}^{\text {th }}$ layer of soil within the root zone $(\mathrm{mm})$; and Asi $=$ apparent specific gravity of the $\mathrm{i}^{\text {th }}$ layer of the soil.

The WUE was calculated by dividing the grain yield of an individual crop by $\mathrm{CW}$ of the respective crop.

\subsection{Crop and System Nutrient Uptake}

Plant samples of grains/seeds/green-pods as well as stalk/stover/straw of different crops collected at harvesting were dried in an oven at $60{ }^{\circ} \mathrm{C}$ for a minimum of 48 hours. The oven-dried samples were ground to pass through 40 mesh-sieve in a Macro-Wiley Mill. From each treatment, grain and byproduct samples were taken for chemical analysis to determine $\mathrm{N}, \mathrm{P}$, and $\mathrm{K}$ concentrations. We estimated $\mathrm{N}$ concentration by the modified Kjeldhal method, P concentration by the Vanado-molybdo-phosphoric yellow color method, and K concentration by the Flame Photometer method following the procedure described by Prasad et al. (2006). The uptake of macro nutrients by each crop was computed by multiplying the $\mathrm{N}, \mathrm{P}$, and $\mathrm{K}$ concentrations with the dry weight of the respective plant parts (grain plus by-product) of each crop at harvest.

The system uptake of $\mathrm{N}, \mathrm{P}$, and $\mathrm{K}$ was estimated by adding nutrient uptake by the component crops for each of the three rainy season cropping systems. A nutrient balance sheet was prepared based on inputs, outputs, and net change in nutrient status before and after the study periods. Apparent nutrient balance was determined based on the total nutrient inputs (initial soil nutrients + nutrients added through recommended nutrients, crop residues, Leucaena twigs, and estimated biological $\mathrm{N}_{2}$ fixation by different legumes) and nutrient outputs (available soil nutrients after harvesting + nutrient uptake both by economic and by-product yields) of all crops and cropping systems. Nutrient balance was estimated by considering the total amount of nutrients added to the soil as different nutrient management options and the total amount of nutrient uptake by grain and straw yields in each year after harvesting the respective crop. This 
calculation was valid particularly for $\mathrm{N}, \mathrm{P}$, and $\mathrm{K}$. The annual nutrient balance $\left(\mathrm{kg} \mathrm{ha}^{-1} \mathrm{y}^{-1}\right)$ was calculated using the following equation (BARC, 2012):

$X_{a}=\left(X_{a}+X_{b}+X_{c r i}\right)-X_{\text {rem }}$

where $\mathrm{X}_{\mathrm{a}}=$ gain or loss of nutrient $\left(\mathrm{kg} \mathrm{ha}^{-1}\right) ; \mathrm{X}_{\mathrm{f}}=$ nutrient added through inorganic sources $\left(\mathrm{kg} \mathrm{ha}^{-1}\right) ; \mathrm{X}_{\mathrm{b}}=$ nutrient added through biological nitrogen fixation $\left(\mathrm{BNF}, \mathrm{kg} \mathrm{ha}^{-1}\right) ; \mathrm{X}_{\text {cri }}=$ nutrient added by incorporation of crop residue $\left(\mathrm{kg} \mathrm{ha}^{-1}\right)$; and $\mathrm{X}_{\mathrm{rem}}=$ nutrient removed by cropping system $\left(\mathrm{kg} \mathrm{ha}^{-}\right.$ $\left.{ }^{1}\right)$. In addition, we also considered initial soil nutrients before planting and available soil nutrients after harvesting in the nutrient balance.

\subsection{Energy Analysis}

Total and net energy inputs from various input sources and outputs from grain and by-products were calculated using the published energy conversion coefficients, and expressed as input energy, output energy, and energy-use efficiency (EUE) (Devasenapathy, SenthilKumar \& Shanmugam, 2009). An inventory of all inputs (e.g., fertilizers, seeds, pesticides, fuel, and human labor) to and outputs (e.g., grain and straw/stover) from all cropping systems was prepared, from which the energy values for each crop management treatment were calculated. Crop inputs and outputs were converted to energy-unit equivalents using conversion coefficients from the published literature to facilitate comparisons among treatments (Tables S1 and S2). The labor and fuel required for each farm operation (e.g., tillage, fertilizer and pesticide applications, hand-weeding, harvesting, and threshing) were recorded for each field trial. Energy outputs were calculated for both economic yield (e.g., sellable harvested product) and straw/stover yield which is used as animal feed on farms. The total energy use (TEU; total energy required to produce a crop), energy output (EO; energy produced in grain and straw products), and EUE were calculated using the following equations (Gathala et al., 2016):

$$
T E U=\left[E_{m}+E_{f}+E_{i}\right]
$$

where TEU $=$ total energy use $\left(\mathrm{MJ} \mathrm{ha}^{-1}\right) ; \mathrm{E}_{m}=$ manual energy use from labor (in person-hours); $\mathrm{E}_{f}=$ the energy used for fuel; and $\mathrm{E}_{i}=$ the energy derived from all inputs (i.e., seed, fertilizer, agro-chemicals, and crop residues). The energy-equivalent factors used in this study are shown in Tables S1 and S2.

$E O=[($ Grain $x$ Energy $)+($ Straw $x$ Energy $)]$

where $\mathrm{EO}=$ energy output $\left(\mathrm{MJ} \mathrm{ha}^{-1}\right)$; grain = crop grain yield $\left(\mathrm{kg} \mathrm{ha}^{-1}\right)$; energy $=$ specific conversion factor for grain or straw $\left(\mathrm{MJ} \mathrm{kg}^{-1}\right)$; and straw $=$ crop straw or stover yield $\left(\mathrm{kg} \mathrm{ha}^{-1}\right)$.

$E U E=\frac{E O}{T E U}$

where EUE = energy-use efficiency (a dimensionless term); EO = energy output $\left(\mathrm{MJ} \mathrm{ha}^{-1}\right)$; and TEU $=$ total energy use $\left(\mathrm{MJ} \mathrm{ha}^{-1}\right)$.

The energy inputs included both renewable (e.g., labor, seed, and crop residues) and non-renewable (e.g., chemical fertilizers, tractor, diesel, machinery, and agro-chemicals) sources of energy.

\subsection{Statistical Analysis}

The data on system comparisons were analyzed using the analysis of variance (ANOVA) with RCBD for 2010 rainy season crops [only three crops (pearl millet, cluster bean, and green gram) under three crop residue retention treatments (no residue, crop residues and Leucaena twigs)]. For the succeeding season's crops that followed the main season crop, the experimental design to analyze ANOVA was strip-split plot design. Thus, cropping systems productivity, EUE, and most of the statistical results were analyzed for the strip-split plot design. The statistical analysis was performed using the MSTAT-C software (Gomez and Gomez, 1984). Least significant difference (LSD) was calculated and treatment means were separated at $5 \%$ level of significance $(\mathrm{P}=0.05)$.

\section{Results and Discussion}

\subsection{Effect of Residue Management on Consumptive Water Use and Water-use Efficiency}

Crop residue management significantly increased both CW and WUE for all cropping systems in both years (Table 1). In most cases, $\mathrm{CW}$ was higher under crop residues than under Leucaena twigs treatment, but the result was inconsistent for WUE between crop residues and Leucaena twigs treatments. Retention of crop residues in pearl millet-based and green gram-based systems, and incorporation of Leucaena twigs in the cluster bean-based system resulted in higher CW and WUE, while no-retention of residues resulted in lower CW and WUE in all cropping systems. The good growth of mustard, chickpea, and wheat after cluster bean, and large amount of cluster bean green-pods resulted in substantially higher $\mathrm{CW}$ and WUE in cluster bean-based cropping systems as compared to pearl milletand green gram-based cropping systems. The $\mathrm{CW}$ was higher in the cluster bean-based system than the other two systems due to its longer crop duration as well, which received more rainfall and had access to soil moisture at deeper depths due to deeper root systems. Increased SOC and improved soil physicochemical and microbiological properties due to the addition of a large amount of crop residues helped increase the retention of more soil moisture (data not shown). Similar to our findings, other studies also reported an improvement in soil structure, water retention capacity, infiltration rate, and hydraulic conductivity, and a decrease in bulk density with the retention or incorporation of crop residues (Edmeades, 2003; Jat et al., 2014). The presence of higher amounts of organic components in leguminous crops, and the applications of both crop residue and Leucaena twigs substantially increased water retention. Large numbers of storage pores in NT residue-applied plots may have resulted in higher soil moisture content at all depths (Azooz, Arshad \& Franzluebbers, 1996). The enhanced soil moisture in the residue-retained NT plots can be attributed to reduced runoff and evaporation as well as greater infiltration (Pingali, Vignozzi \& Pellegrini, 2004; Verhulst, Deckers \& Govaerts, 2009). As a result, CW was substantially higher in residue-applied plots. Similar results were reported by several studies (Chaudhari, 1999; Gathala et al., 2011b, 2016; Saharawat et al., 2010; Singh \& Singh, 1995) that increased root activity and proliferation of root system due to translocation of more photosynthates to roots that resulted in more extraction of soil moisture from deeper layers $(>15 \mathrm{~cm})$. 
Table 1. Effect of crop residues and Leucaena twigs on consumptive water use $(\mathrm{CW}, \mathrm{mm})$ and water-use efficiency $\left(\mathrm{WUE}, \mathrm{kg} \mathrm{ha}^{-1} \mathrm{~mm}^{-1}\right)$ of pearl millet-, cluster bean-, and green gram-based cropping systems.

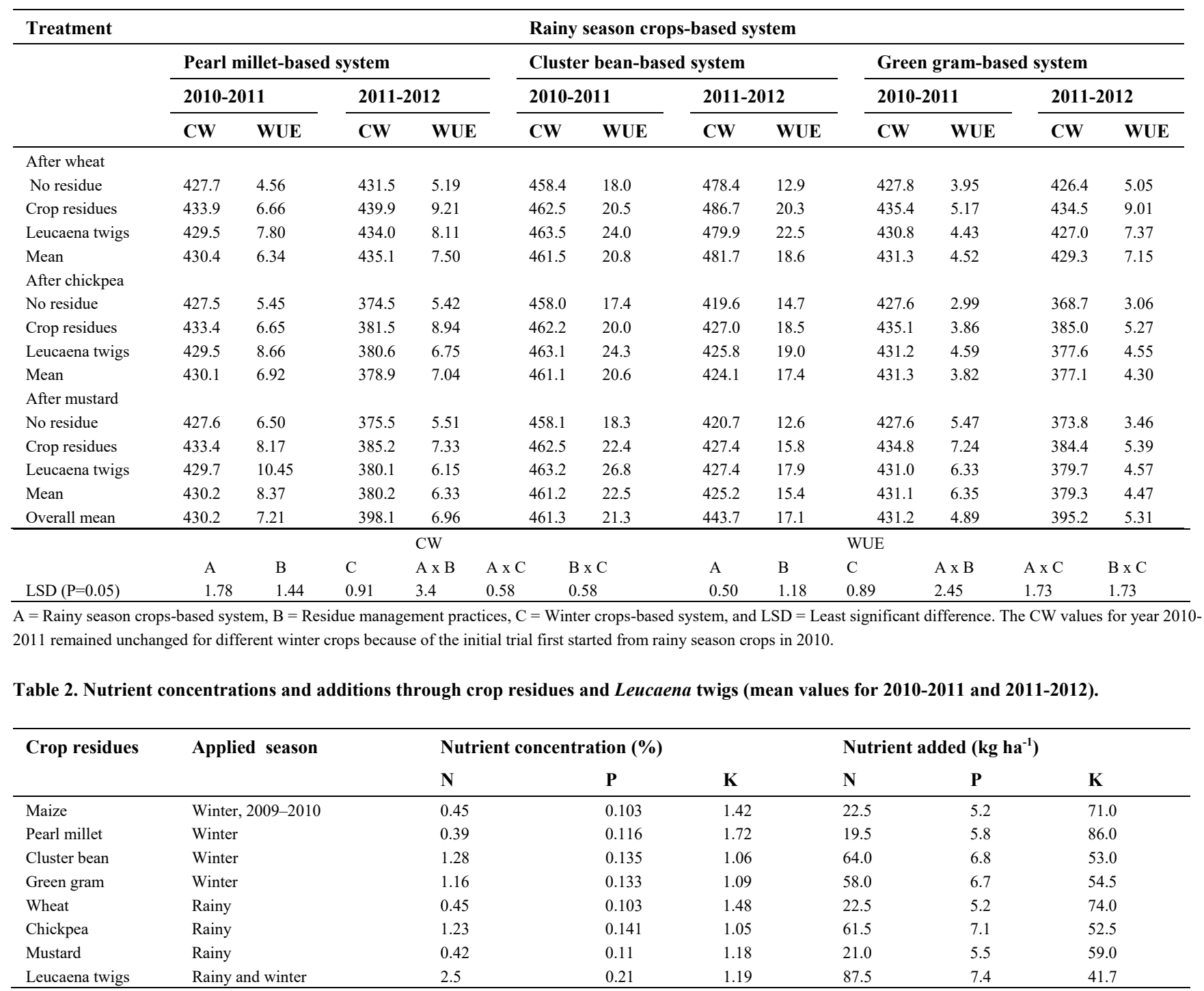

Crop residues were applied at $5.0 \mathrm{t}$ ha- 1 dry matter and Leucaena twigs were applied at $10.0 \mathrm{t}$ ha-1 green twigs ( $\sim 3.5 \mathrm{t}$ ha-1 dry matter). 
Table 3. System uptake of nitrogen $\left(\mathrm{N}, \mathrm{kg} \mathrm{ha}^{-1}\right)$ as influenced by residue management.

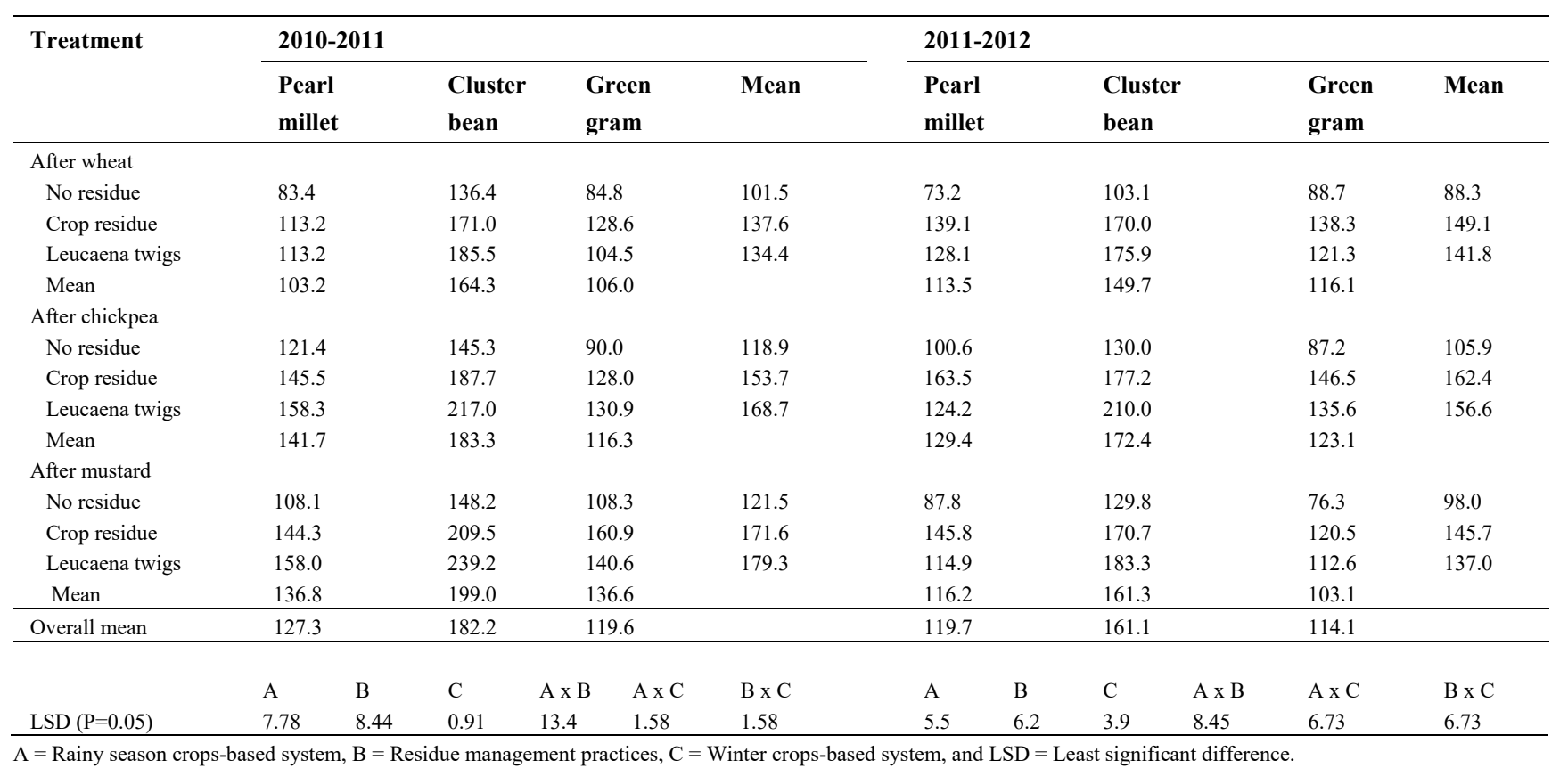

Table 4. System uptake of phosphorus $\left(\mathrm{P}, \mathrm{kg} \mathrm{ha}^{-1}\right)$ as influenced by residue management.

\begin{tabular}{|c|c|c|c|c|c|c|c|c|c|c|c|c|}
\hline \multirow[t]{2}{*}{ Treatment } & \multicolumn{6}{|c|}{$2010-2011$} & \multicolumn{6}{|c|}{ 2011-2012 } \\
\hline & \multicolumn{2}{|c|}{$\begin{array}{l}\text { Pearl } \\
\text { millet }\end{array}$} & \multicolumn{2}{|l|}{$\begin{array}{l}\text { Cluster } \\
\text { bean }\end{array}$} & \multirow[t]{2}{*}{$\begin{array}{l}\text { Green } \\
\text { gram }\end{array}$} & Mean & \multicolumn{2}{|c|}{$\begin{array}{l}\text { Pearl } \\
\text { millet }\end{array}$} & \multicolumn{2}{|l|}{$\begin{array}{l}\text { Cluster } \\
\text { bean }\end{array}$} & $\begin{array}{l}\text { Green } \\
\text { gram }\end{array}$ & Mean \\
\hline \multicolumn{12}{|l|}{ After wheat } & \\
\hline No residue & 21.9 & & 18.4 & & 13.1 & 17.8 & 20.1 & & 14.7 & & 15.4 & 16.7 \\
\hline Crop residue & 29.2 & & 24.2 & & 20.0 & 24.5 & 35.1 & & 26.4 & & 25.2 & 28.9 \\
\hline Leucaena twigs & 29.2 & & 26.1 & & 16.2 & 23.8 & 34.3 & & 28.6 & & 20.8 & 27.9 \\
\hline Mean & 26.7 & & 22.9 & & 16.4 & & 29.8 & & 23.2 & & 20.4 & \\
\hline \multicolumn{13}{|l|}{ After chickpea } \\
\hline No residue & 23.5 & & 16.9 & & 9.8 & 16.7 & 22.1 & & 14.5 & & 9.3 & 15.3 \\
\hline Crop residue & 28.2 & & 20.9 & & 13.7 & 21.0 & 30.9 & & 18.5 & & 15.2 & 21.5 \\
\hline Leucaena twigs & 29.2 & & 24.5 & & 13.3 & 22.3 & 25.7 & & 22.4 & & 14.3 & 20.8 \\
\hline Mean & 27.0 & & 20.8 & & 12.2 & & 26.3 & & 18.4 & & 12.9 & \\
\hline \multicolumn{13}{|l|}{ After mustard } \\
\hline No residue & 26.7 & & 21.7 & & 17.2 & 21.9 & 25.4 & & 18.3 & & 11.8 & 18.5 \\
\hline Crop residue & 36.3 & & 32.2 & & 26.5 & 31.7 & 41.2 & & 26.2 & & 21.8 & 29.8 \\
\hline Leucaena twigs & 38.0 & & 36.7 & & 23.6 & 32.8 & 33.8 & & 27.3 & & 19.0 & 26.7 \\
\hline Mean & 33.7 & & 30.2 & & 22.4 & & 33.5 & & 24.0 & & 17.5 & \\
\hline \multirow[t]{2}{*}{ Overall mean } & 29.1 & & 24.6 & & 17.0 & & 29.9 & & 21.9 & & 17.0 & \\
\hline & A & B & $\mathrm{C}$ & $\mathrm{A} \times \mathrm{B}$ & $\mathrm{A} \times \mathrm{C}$ & $\mathrm{B} \times \mathrm{C}$ & A & B & $\mathrm{C}$ & $\mathrm{A} \times \mathrm{B}$ & $\mathrm{A} \times \mathrm{C}$ & $\mathrm{B} \times \mathrm{C}$ \\
\hline $\operatorname{LSD}(\mathrm{P}=0.05)$ & 1.2 & 1.07 & 0.34 & 1.8 & 0.58 & 0.58 & 1.63 & 1.41 & 1.12 & 2.09 & 1.95 & 1.95 \\
\hline
\end{tabular}

$\mathrm{A}=$ Rainy season crops-based system, $\mathrm{B}=$ Residue management practices, $\mathrm{C}=$ Winter crops-based system, and LSD $=$ Least significant difference. 
Table 5. System uptake of potassium $\left(\mathrm{K}, \mathrm{kg} \mathrm{ha}^{-1}\right)$ as influenced by residue management.

\begin{tabular}{|c|c|c|c|c|c|c|c|c|c|c|c|c|}
\hline \multirow[t]{2}{*}{ Treatment } & \multicolumn{6}{|c|}{ 2010-2011 } & \multicolumn{6}{|c|}{ 2011-2012 } \\
\hline & $\begin{array}{l}\text { Pearl } \\
\text { millet }\end{array}$ & & $\begin{array}{l}\text { Cluster } \\
\text { bean }\end{array}$ & & $\begin{array}{l}\text { Green } \\
\text { gram }\end{array}$ & Mean & $\begin{array}{l}\text { Pearl } \\
\text { millet }\end{array}$ & & $\begin{array}{l}\text { Cluster } \\
\text { bean }\end{array}$ & & $\begin{array}{l}\text { Green } \\
\text { gram }\end{array}$ & Mean \\
\hline \multicolumn{13}{|l|}{ After wheat } \\
\hline No residue & 130.8 & & 93.0 & & 91.9 & 105.2 & 96. & & 67.7 & & 82.0 & 82.2 \\
\hline Crop residue & 188.5 & & 135.0 & & 154.6 & 159.4 & 191 & & 118.4 & & 133.2 & 147.5 \\
\hline Leucaena twigs & 168.8 & & 133.8 & & 113.5 & 138.7 & 178 & & 122.7 & & 110.9 & 137.3 \\
\hline Mean & 162.7 & & 120.6 & & 120.0 & & 155 & & 102.9 & & 108.7 & \\
\hline \multicolumn{13}{|l|}{ After chickpea } \\
\hline No residue & 139.6 & & 85.6 & & 67.3 & 97.5 & 119 & & 77.2 & & 64.0 & 87.0 \\
\hline Crop residue & 174.8 & & 104.6 & & 91.3 & 123.6 & 182 & & 104.0 & & 102.6 & 129.6 \\
\hline Leucaena twigs & 161.4 & & 117.3 & & 83.1 & 120.6 & 145 & & 130.4 & & 99.5 & 125.3 \\
\hline Mean & 158.6 & & 102.5 & & 80.6 & & 149 & & 103.9 & & 88.7 & \\
\hline \multicolumn{13}{|l|}{ After mustard } \\
\hline No residue & 134.9 & & 99.3 & & 89.5 & 107.9 & 124 & & 95.9 & & 63.7 & 94.6 \\
\hline Crop residue & 202.6 & & 153.2 & & 143.0 & 166.3 & 222 & & 142.9 & & 122.4 & 162.5 \\
\hline Leucaena twigs & 191.5 & & 173.1 & & 126.0 & 163.5 & 177 & & 142.8 & & 107.7 & 142.6 \\
\hline Mean & 176.3 & & 141.9 & & 119.5 & & 174 & & 127.2 & & 98.0 & \\
\hline \multirow[t]{2}{*}{ Overall mean } & 165.9 & & 121.7 & & 106.7 & & 159 & & 111.3 & & 98.4 & \\
\hline & A & B & $\mathrm{C}$ & $A \times B$ & $\mathrm{~A} \times \mathrm{C}$ & $\mathrm{B} \times \mathrm{C}$ & A & B & $\mathrm{C}$ & $A \times B$ & $\mathrm{~A} \times \mathrm{C}$ & $\mathrm{B} \times \mathrm{C}$ \\
\hline $\operatorname{LSD}(\mathrm{P}=0.05)$ & 10.3 & 7.21 & 2.47 & 7.21 & 4.28 & 4.28 & 10.0 & 7.41 & 6.14 & 9.68 & 10.6 & 10.6 \\
\hline
\end{tabular}

$\mathrm{A}=$ Rainy season crops-based system, $\mathrm{B}=$ Residue management practices, $\mathrm{C}=$ Winter crops-based system, and LSD $=$ Least significant difference.

Table 6. Total input energy (MJ ha-1) expended for cultivation of rainy season and winter season crops under no till with residues management (mean values for 2010-2011 and 2011-2012).

\begin{tabular}{|c|c|c|c|c|c|c|c|}
\hline Particulars & Common energy & Variable energy & Total energy & Particulars & Common energy & Variable energy & Total energy \\
\hline A. Rainy season crops & & & & B. Winter season & & & \\
\hline 1. Pearl millet & & & & 1. Wheat & & & \\
\hline No residues & 5812 & 0 & 5812 & No residues & 7534 & 0 & 7534 \\
\hline Crop residues & 5812 & 62547 & 68359 & Crop residues & 7534 & 62547 & 70081 \\
\hline Leucaena twigs & 5812 & 43906 & 49719 & Leucaena twigs & 7534 & 43906 & 51440 \\
\hline 2. Cluster bean & & & & 2. Chickpea & & & \\
\hline No residues & 3791 & 0 & 3791 & No residues & 4447 & 0 & 4447 \\
\hline Crop residues & 3791 & 62547 & 66368 & Crop residues & 4447 & 62547 & 66994 \\
\hline Leucaena twigs & 3791 & 43906 & 47698 & Leucaena twigs & 4447 & 43907 & 48353 \\
\hline 3. Green gram & & & & 3. Mustard & & & \\
\hline No residues & 3907 & 0 & 3907 & No residues & 5857 & 0 & 5857 \\
\hline Crop residues & 3907 & 62547 & 66454 & Crop residues & 5857 & 62547 & 68404 \\
\hline Leucaena twigs & 3907 & 43907 & 47813 & Leucaena twigs & 5857 & 43907 & 49764 \\
\hline
\end{tabular}




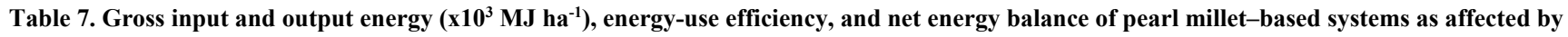
residue management.

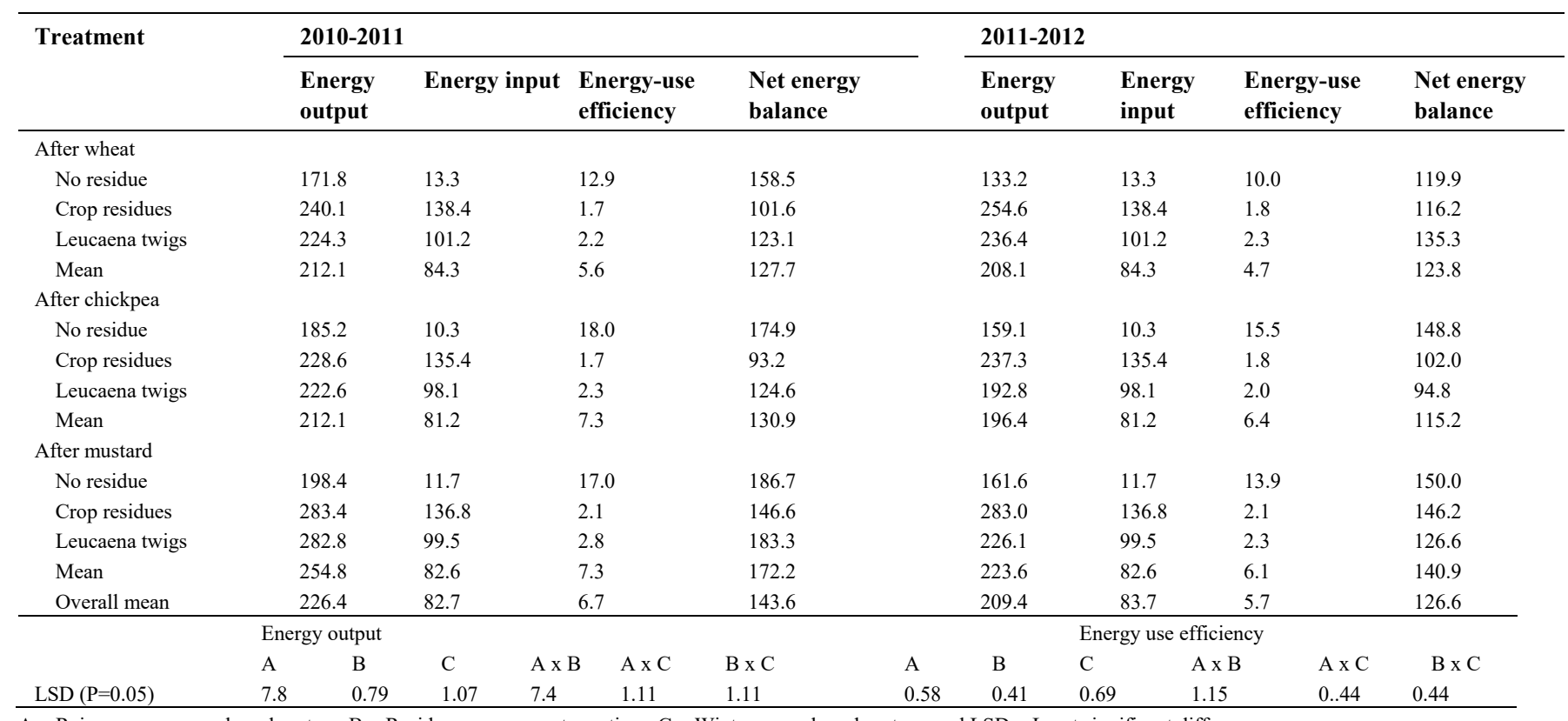

$\mathrm{A}=$ Rainy season crops-based system, $\mathrm{B}=$ Residue management practices, $\mathrm{C}=$ Winter crops-based system, and LSD $=$ Least significant difference.

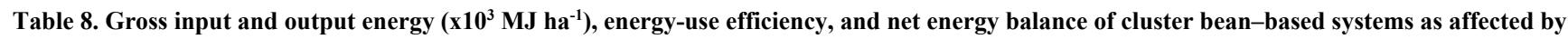
residue management.

\begin{tabular}{|c|c|c|c|c|c|c|c|c|c|c|c|c|}
\hline \multirow[t]{2}{*}{ Treatment } & \multicolumn{6}{|c|}{ 2010-2011 } & \multicolumn{6}{|c|}{ 2011-2012 } \\
\hline & \multicolumn{2}{|c|}{$\begin{array}{l}\text { Energy } \\
\text { output }\end{array}$} & $\begin{array}{l}\text { Energy } \\
\text { input }\end{array}$ & \multicolumn{2}{|c|}{$\begin{array}{l}\text { Energy use } \\
\text { efficiency }\end{array}$} & $\begin{array}{l}\text { Net energy } \\
\text { balance }\end{array}$ & \multicolumn{2}{|l|}{$\begin{array}{l}\text { Energy } \\
\text { output }\end{array}$} & $\begin{array}{l}\text { Energy } \\
\text { input }\end{array}$ & \multicolumn{2}{|c|}{$\begin{array}{l}\text { Energy use } \\
\text { efficiency }\end{array}$} & $\begin{array}{l}\text { Net energy } \\
\text { balance }\end{array}$ \\
\hline \multicolumn{13}{|l|}{ After wheat } \\
\hline No residue & 127.6 & & 11.3 & 11.3 & & 116.3 & 92.2 & & 11.3 & 8.1 & & 80.9 \\
\hline Crop residues & 179.1 & & 136.4 & 1.3 & & 42.7 & 166.9 & & 136.4 & 1.2 & & 30.4 \\
\hline Leucaena twigs & 180.8 & & 99.1 & 1.8 & & 81.7 & 173.9 & & 99.1 & 1.8 & & 74.7 \\
\hline Mean & 162.5 & & 82.3 & 4.8 & & 80.2 & 144.3 & & 82.3 & 3.7 & & 62.0 \\
\hline \multicolumn{13}{|l|}{ After chickpea } \\
\hline No residue & 118.6 & & 8.2 & 14.4 & & 110.4 & 107.8 & & 8.2 & 13.1 & & 99.5 \\
\hline Crop residues & 148.9 & & 133.3 & 1.1 & & 15.6 & 146.1 & & 133.3 & 1.1 & & 12.8 \\
\hline Leucaena twigs & 167.3 & & 96.1 & 1.7 & & 71.2 & 178.2 & & 96.1 & 1.9 & & 82.1 \\
\hline Mean & 144.9 & & 79.2 & 5.8 & & 65.7 & 144.0 & & 79.2 & 5.3 & & 64.8 \\
\hline \multicolumn{13}{|l|}{ After mustard } \\
\hline No residue & 144.3 & & 9.6 & 15.0 & & 134.6 & 130.8 & & 9.6 & 13.6 & & 121.1 \\
\hline Crop residues & 229.4 & & 134.7 & 1.7 & & 94.6 & 189.3 & & 134.7 & 1.4 & & 54.6 \\
\hline Leucaena twigs & 258.4 & & 97.5 & 2.7 & & 160.9 & 200.7 & & 97.5 & 2.1 & & 103.2 \\
\hline Mean & 210.7 & & 80.6 & 6.4 & & 130.1 & 173.6 & & 80.6 & 5.7 & & 93.0 \\
\hline \multirow[t]{3}{*}{ Overall mean } & 172.7 & & 80.7 & 5.7 & & 92.0 & 154.0 & & 80.7 & 4.9 & & 73.3 \\
\hline & \multicolumn{6}{|c|}{ Energy output } & \multicolumn{6}{|c|}{ Energy use efficiency } \\
\hline & A & B & $\mathrm{C}$ & $\mathrm{A} \times \mathrm{B}$ & $\mathrm{A} \times \mathrm{C}$ & $\mathrm{B} \times \mathrm{C}$ & A & B & $\mathrm{C}$ & $\mathrm{A} \times \mathrm{B}$ & $\mathrm{A} \times \mathrm{C}$ & $\mathrm{B} \times \mathrm{C}$ \\
\hline $\operatorname{LSD}(\mathrm{P}=0.05)$ & 2.3 & 0.37 & 0.41 & 1.78 & 0.76 & 0.76 & 0.28 & 0.22 & 0.35 & 0.73 & 0.23 & 0.23 \\
\hline
\end{tabular}

$\mathrm{A}=$ Rainy season crops-based system, $\mathrm{B}=$ Residue management practices, $\mathrm{C}=$ Winter crops-based system, and $\mathrm{LSD}=\mathrm{Least}$ significant difference. 
Table 9. Gross input and output energy $\left(\mathrm{x}^{10^{3}} \mathrm{MJ} \mathrm{ha}^{-1}\right)$, energy-use efficiency, and net energy balance of green gram-based systems as affected by residue management.

\begin{tabular}{|c|c|c|c|c|c|c|c|c|c|c|c|c|}
\hline \multirow[t]{2}{*}{ 'Treatment } & \multicolumn{6}{|c|}{ 2010-2011 } & \multicolumn{6}{|c|}{ 2011-2012 } \\
\hline & \multicolumn{2}{|c|}{$\begin{array}{l}\text { Energy } \\
\text { output }\end{array}$} & $\begin{array}{l}\text { Energy } \\
\text { input }\end{array}$ & \multicolumn{2}{|c|}{$\begin{array}{l}\text { Energy- use } \\
\text { efficiency }\end{array}$} & $\begin{array}{l}\text { Net energy } \\
\text { balance }\end{array}$ & \multicolumn{2}{|c|}{$\begin{array}{l}\text { Energy } \\
\text { output }\end{array}$} & Energy input & \multicolumn{2}{|l|}{$\begin{array}{l}\text { Energy- use } \\
\text { efficiency }\end{array}$} & $\begin{array}{l}\text { Net energy } \\
\text { balance }\end{array}$ \\
\hline \multicolumn{13}{|l|}{ After wheat } \\
\hline No residue & & & 11.4 & 9.9 & & 102.1 & 109.5 & & 11.4 & 9.6 & & 98.1 \\
\hline Crop residues & & & 136.5 & 1.4 & & 53.2 & 181.4 & & 136.5 & 1.3 & & 44.9 \\
\hline Leucaena twigs & & & 99.3 & 1.4 & & 43.2 & 150.1 & & 99.3 & 1.5 & & 50.9 \\
\hline Mean & & & 82.4 & 4.3 & & 66.2 & 147.0 & & 82.4 & 4.1 & & 64.6 \\
\hline \multicolumn{13}{|l|}{ After chickpea } \\
\hline No residue & & & 8.4 & 10.4 & & 78.4 & 88.5 & & 8.4 & 10.6 & & 80.1 \\
\hline Crop residues & & & 133.4 & 0.9 & & -9.6 & 139.2 & & 133.4 & 1.0 & & 5.8 \\
\hline Leucaena twigs & & & 96.2 & 1.2 & & 18.6 & 134.3 & & 96.2 & 1.4 & & 38.1 \\
\hline Mean & & & 79.3 & 4.2 & & 29.1 & 120.7 & & 79.3 & 4.3 & & 41.3 \\
\hline \multicolumn{13}{|l|}{ After mustard } \\
\hline No residue & & & 9.8 & 13.7 & & 123.5 & 88.8 & & 9.8 & 9.1 & & 79.0 \\
\hline Crop residues & & & 134.9 & 1.6 & & 77.2 & 165.5 & & 134.9 & 1.2 & & 30.7 \\
\hline Leucaena twigs & & & 97.6 & 1.9 & & 86.8 & 144.2 & & 97.6 & 1.5 & & 46.7 \\
\hline Mean & & & 80.7 & 5.7 & & 95.8 & 132.8 & & 80.7 & 3.9 & & 52.1 \\
\hline \multirow[t]{3}{*}{ Overall mean } & & & 80.8 & 4.7 & & 63.7 & 133.5 & & 80.8 & 4.1 & & 52.7 \\
\hline & \multicolumn{6}{|c|}{ Energy output } & \multicolumn{6}{|c|}{ Energy use efficiency } \\
\hline & A & $\mathrm{B}$ & $\mathrm{C}$ & $\mathrm{A} \times \mathrm{B}$ & $\mathrm{A} \times \mathrm{C}$ & $\mathrm{B} \times \mathrm{C}$ & A & B & $\mathrm{C}$ & $\mathrm{A} \times \mathrm{B}$ & $\mathrm{A} \times \mathrm{C}$ & $\mathrm{B} \times \mathrm{C}$ \\
\hline $\operatorname{LSD}(\mathrm{P}=0.05)$ & 1.4 & 0.29 & 0.32 & 1.17 & 0.43 & 0.43 & 0.19 & $0 . .14$ & 0.29 & 0.51 & $0 . .17$ & 0.17 \\
\hline
\end{tabular}

$\mathrm{A}=$ Rainy season crops-based system, $\mathrm{B}=$ Residue management practices, $\mathrm{C}=$ Winter crops-based system, and LSD $=$ Least significant difference.

Due to several advantages associated with the application of crop residues, it can improve crop yields (Jin \& Yibing, 2001). Significantly higher pearl millet-equivalent yield was obtained for cluster bean after wheat and chickpea under Leucaena twigs, followed by residue retention than other rainy season crops (pearl millet and green gram). Significantly higher wheat-equivalent yields $\left(4.15 \mathrm{t} \mathrm{ha}^{-1}\right.$ in 2010-2011 and $3.77 \mathrm{t} \mathrm{ha}^{-1}$ in 2011-2012) were obtained for mustard under Leucaena twigs after cluster bean. Although both yield and $\mathrm{CW}$ increased under residue treatments, more increase in yield than the corresponding increase in CW by applying residues resulted in higher WUE. These results were consistent with the results from previous studies (Gathala et al., 2013; Wanga et al., 2010). Another study also reported that incorporation of crop residues increased WUE by $10-20 \%$ in arid and semi-arid regions of China (Deng, Shan, Zhang \& Turner, 2006).

\subsection{Effect of Residue Management on System Level Nutrient Uptake and Balances}

The N, $\mathrm{P}$, and $\mathrm{K}$ concentrations and nutrient additions through crop residues and Leucaena twigs had substantial variations among crops due to differences in nutritional contents of the residues (Table 2). System uptake of N (Table 3), P (Table 4), and K (Table 5), and apparent nutrient balances (Figures 2, 3, and 4) revealed that the residue retention of the preceding crops resulted in significant variations in uptake and balances of $\mathrm{N}, \mathrm{P}$, and $\mathrm{K}$ for the cropping systems. The actual balances of different nutrients varied widely across treatments because of the addition of recommended doses of fertilizers along with the crop residues, Leucaena twigs, and variable quantities of biomass through leaf litter, root, and nodule biomass of legumes, as well as root and stubbles of wheat, pearl millet, and mustard.

There were smaller uptakes of $\mathrm{N}, \mathrm{P}$, and $\mathrm{K}$ for all cropping systems under no-residue control plots (Tables 3, 4, and 5). No-residue plots received only the blanket dose of recommended $\mathrm{N}, \mathrm{P}$, and $\mathrm{K}$, and hence the system uptake was less because of reduced biomass production and less availability of nutrients. Uptake of $\mathrm{N}, \mathrm{P}$, and $\mathrm{K}$ increased substantially under crop residue and Leucaena twigs treatments due to enhanced aboveground plant growth and root growth, and addition of nutrients. In general, as compared to crop residue treatment, uptake of $\mathrm{N}, \mathrm{P}$, and $\mathrm{K}$ increased for the cluster bean-based system and decreased for the green gram-based system under the Leucaena twigs treatment (Tables 3, 4, and 5). However, for the pearl millet-based system, $N$ and $P$ uptakes were higher in 2010-2011 but lower in 2011-2012 under Leucaena twigs treatment as compared to crop residue treatment. Uptake of $\mathrm{K}$ was higher under crop residue than Leucaena twigs treatment for the pearl millet-based system in both years (Table 5).

Crop residue application added more $\mathrm{K}$ to the systems, while Leucaena twigs added more $\mathrm{N}$ and $\mathrm{P}$ (Table 2). The good growth of mustard, chickpea, and wheat after cluster bean, and large amounts of cluster bean green-pods resulted in substantially higher uptake of $\mathrm{N}$ in cluster beanbased systems as compared to pearl millet- and green gram-based systems. Pearl millet-based systems had higher uptake of $\mathrm{P}$ and $\mathrm{K}$ than the other two systems because of the higher biomass produced by the pearl millet-based system in comparison to cluster bean and green gram-based systems. Consistent with our findings, previous studies have also documented higher nutrient uptake with crop residue incorporation in pearl millet (Das \& Gautam, 2003; Sarker, Patra, Mula \& Paramanik, 2011; Vyas, Patel, Patel \& Khanpara, 1994; Yadav, Kumar \& Kumar, 2009), cluster bean (Buttar, Thind, Saroa \& Grover, 2009; Solanki \& Sahu, 2007), and green grambased systems (Singh et al., 2008). The similar findings have been recorded for the rice-maize rotations in South Asia (Kumar et al., 2013a; Singh, Singh \& Timsina, 2005, Timsina, Jat \& Majumdar, 2010).

The apparent $\mathrm{N}, \mathrm{P}$, and $\mathrm{K}$ balances for various cropping systems were mostly positive for $\mathrm{N}$ and $\mathrm{P}$, and negative for $\mathrm{K}$ at the end of two years of the experiment, more likely due to low initial status of $\mathrm{K}$ fertility and more uptake of $\mathrm{K}$ by the cereal-based systems (Figures 2, 3, and 4). Legumes like chickpea, cluster bean, and green gram fix atmospheric $\mathrm{N}_{2}$ and help increase the input of $\mathrm{N}$ under legume-based systems. In addition, we had applied the recommended dose of $20 \mathrm{~kg} \mathrm{~N}^{-1}$ to legumes and $60 \mathrm{~kg} \mathrm{~N} \mathrm{ha}^{-1}$ to non- 
legumes (pearl millet, wheat, and mustard) as suggested by Reddy \& Reddi (2009).

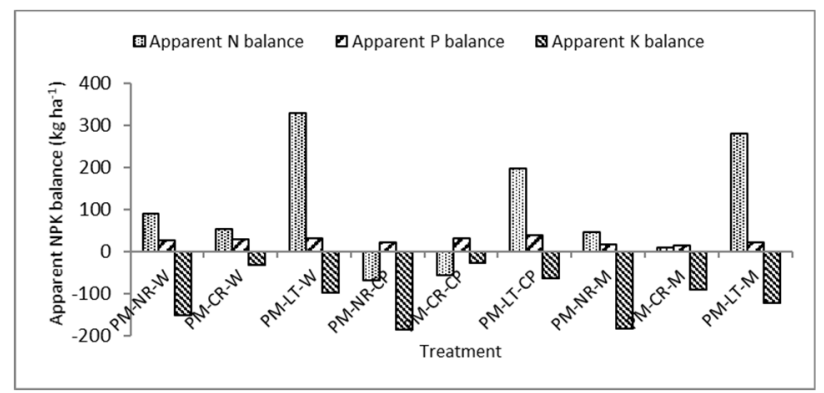

Figure 2. Apparent balance of nitrogen (N), phosphorus (P), and potassium $(\mathrm{K})$ in pearl millet-based systems as influenced by residue management after two years of experiment.

Note: $\mathrm{PM}=$ Pearl millet, $\mathrm{NR}=$ No residue, $\mathrm{CR}=$ Crop residue, $\mathrm{LT}=$ Leucaena twigs, $\mathrm{W}=$ Wheat, $\mathrm{CP}=$ Chickpea, and $\mathrm{M}=$ Mustard.

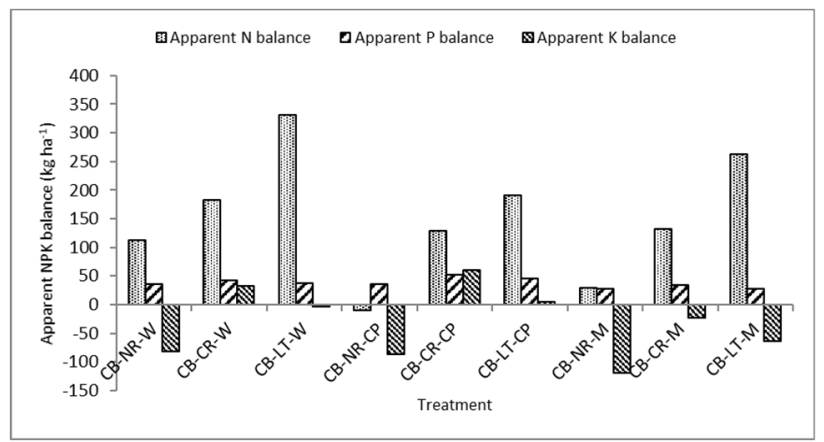

Figure 3. Apparent balance of nitrogen (N), phosphorus (P), and potassium $(\mathrm{K})$ in cluster bean -based systems as influenced by residue management after two years of experiment.

Note: $\mathrm{CB}=$ Cluster bean, $\mathrm{NR}=$ No residue, $\mathrm{CR}=$ Crop residue, $\mathrm{LT}=$ Leucaena twigs, $\mathrm{W}=$ Wheat, $\mathrm{CP}=$ Chickpea, and $\mathrm{M}=$ Mustard.

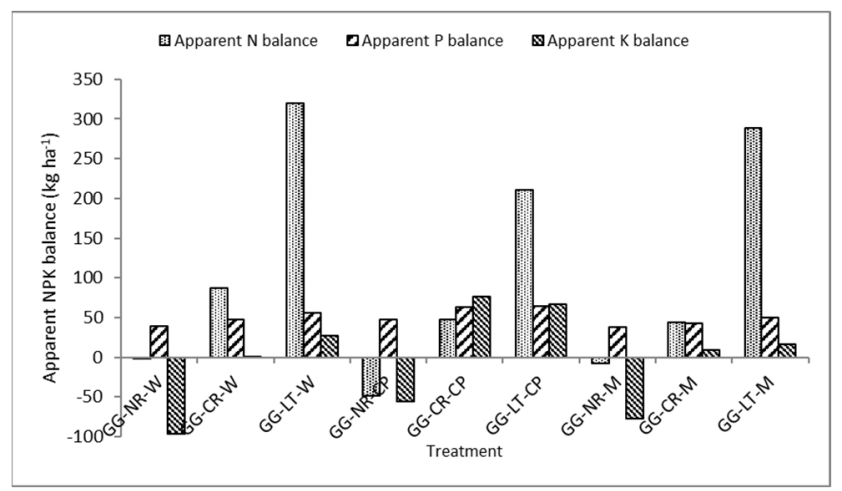

Figure 4. Apparent balance of nitrogen (N), phosphorus (P), and potassium $(\mathrm{K})$ in green gram-based systems as influenced by residue management after two years of experiment.

Note: $\mathrm{GG}=$ Green gram, $\mathrm{NR}=$ No residue, $\mathrm{CR}=$ Crop residue, $\mathrm{LT}=$ Leucaena twigs, $\mathrm{W}=$ Wheat, $\mathrm{CP}=$ Chickpea, and $\mathrm{M}=$ Mustard.

\subsection{Effect of Residue Management on Crop Energetics}

Input energy consumptions (both renewable and non-renewable) varied across various residue management practices (Table 6). Residue management increased input energy due to the addition of $5 \mathrm{t} \mathrm{ha}^{-1}$ dry biomass of crop residues and $3.5 \mathrm{t} \mathrm{ha}^{-1}$ dry biomass of Leucaena twigs in all cropping systems (Tables 7, 8, and 9). The common energy sources were fertilizer, seed, labors, and agro-chemicals. Higher variable energy was required to incorporate $5 \mathrm{t} \mathrm{ha}^{-1}$ dry matter of crop residue $(\sim 62,500 \mathrm{MJ}$ ha $\left.{ }^{1}\right)$ than to incorporate $3.5 \mathrm{tha}^{-1}$ dry matter of Leucaena twigs ( 44,000 MJ $\left.\mathrm{ha}^{-1}\right)$. Chaudhary et al. (2006) reported that the high energy value of crop residue (12.5 $\mathrm{MJ} \mathrm{kg}^{-1}$ ) was the reason for the maximum energy requirement by the residue retention treatments. Input energy was higher for crop residue treatment than Leucaena twigs treatment. The input energy requirement under crop residue and Leucaena twigs was about 11 and 9 times higher, respectively, than under no-residue for all cropping systems. The results highlighted the importance of applying leguminous residues in crop production to reduce energy usage.

Gross output energy produced by the pearl millet-based system was higher than cluster bean- and green gram-based systems due to higher biomass of pearl millet than cluster bean and green gram. In 2010, the highest gross output energy was produced under pearl millet after mustard $\left(283.4 \times 10^{3} \mathrm{MJ} \mathrm{ha}^{-1}\right)$ followed by pearl millet after wheat $\left(240.1 \times 10^{3} \mathrm{MJ}\right.$ $\left.\mathrm{ha}^{-1}\right)$, both under crop residue retention. The highest gross output energy under the pearl millet-based system after mustard with residue retention was due to the comparatively higher biomass yield of pearl millet and mustard. Consistent with our findings, Mandal et al. (2002) also reported a higher energy requirement of the pearl millet-based system due to high energy consumed by crop residues and fertilizers. The lowest gross output energy under no residue retention under all three cropping systems was due to their lower yield performance. The treatment with no residue recorded the lowest energy requirement of all cropping systems due to savings of energy that would otherwise be needed with residue retention.

Maximum net energy was recorded under the pearl millet-based system with no residue after mustard $\left(186.7 \times 10^{3} \mathrm{MJ} \mathrm{ha}^{-1}\right)$ followed by chickpea $\left(174.9 \times 10^{3} \mathrm{MJ} \mathrm{ha}^{-1}\right)$ and wheat (158.5 x 10 $\left.0^{3} \mathrm{MJ} \mathrm{ha}^{-1}\right)$ during 2010-2011, while the lowest net energy production was observed under crop residue retention. Maximum EUE was recorded for no-residue treatment after chickpea and mustard (17-18) under the pearl millet-based system in 20102011 than in the other two systems. The EUE was substantially lower under crop residue and Leucaena twigs treatments as compared to no-residue treatment for all cropping systems due to higher energy requirements. Thus, even though yield performance was better under crop residue and Leucaena twigs treatments, they did not improve EUE.

\section{Conclusion}

The study determined the influence of nine cropping systems with conservation agricultural practices on the water and nutrient uptake and balance, energy relations, and resource-use efficiencies under a no-till semi-arid environment of India. The CW and WUE were higher for cluster bean-chickpea and cluster bean-mustard systems under Leucaena twigs and crop residue retention cropping systems. Both CW and WUE were substantially higher for all cropping systems under crop residue and Leucaena twigs treatments as compared to no-residue treatment. Uptakes of nutrients (N, P, and $\mathrm{K}$ ) were smaller for all cropping systems under noresidue treatment, most likely due to reduced productivity and less 
availability of nutrients. Input energy, gross output energy, net output energy, and EUE were relatively higher under the pearl millet-based system than cluster bean-and green gram-based systems due to the higher biomass of pearl millet. As residue management increased the input energy, no-residue treatment showed higher EUE. The results indicated that the retention of crop residues might require more energy input in rainfed cropping systems. Thus, an optimal balance between retention of crop residues and energy inputs might be critical for the long-term sustainability of rainfed cropping systems.

\section{Acknowledgments}

The research article was a part of the Ph.D. thesis of Lal P. Amgain at the Division of Agronomy, Indian Agricultural Research Institute (IARI), Pusa, New Delhi. LPA acknowledges the Indian Council of Cultural Relations and Institute of Agriculture and Animal Science, Rampur, Nepal for granting the South Asian Association for Regional Cooperation (SAARC) fellowship and study leave, respectively, for a Ph.D. program at IARI.

\section{References}

Acharya, C. L., Kapur, O. C., \& Dixit, S. P. (1998). Moisture conservation for rainfed wheat production with alternative mulches and conservation tillage in hills of north-west India. Soil and Tillage Research, 46, 153-163.

Allen, R. G., Pereira, L., Raes, S., \& Smith, D. (1998). Cropevapotranspiration guidelines for computing crop water requirement. FAO Irrigation and Drainage Paper, 56, 27-32.

Aryal, J. P., Sapkota, T. B., Jat, M. L., \& Bishnoi, D. K. (2015). On-farm economic and environmental input of zero-tillage wheat: a case of northwest India. Experimental Agriculture, 51(1), 1-16.

Azooz, R. H., Arshad, M. A., \& Franzluebbers, A. J. (1996). Pore size distribution and hydraulic conductivity affected by tillage in Northwestern Canada. Soil Science Society of America Journal, 60, 1197-1201.

Bandalucco, L., Rao, M., Colombo, C., Palumbo, G., Laudiciana, V. A., \& Gianfreda, L. (2010). Reversing agriculture from intensive to sustainable to improve soil quality in a semi-arid south Italian soil. Biology and Fertility of Soils, 46, 481-489.

BARC (2012). Fertilizer Recommendation Guide-2012. Farmgate, Dhaka1215; BARC: Dhaka, Bangladesh.

Bhusan, L., \& Sharma, P. K. (2002). Long-term effect of Lantana (Lantana sp. L.) residues additions on soil physical properties under rice-wheat cropping. I Soil consistency, surface cracking and clod formation. Soil and Tillage Research, 65, 157-167.

Buttar, G. S., Thind, H. S., Saroa, G. S., \& Grover, K. (2009). Performance of wheat (Triticum aestivum) as influenced by $\mathrm{N}$ fertilization in cluster bean (Cyamopsis tetragonoloba)-wheat (Triticum aestivum) system. Indian Journal of Agricultural Sciences, 79(4), 302-304.

Chaudhary, V. P., Gangwar, B., \& Pandey, D. K. (2006). Auditing of energy use and output of different cropping systems in India. Agricultural Engineering International: CIGR Journal, 8, 652-658.

Chaudhari, A. C. (1999). Response of rainfed pearl millet [Pennisetum glaucum (L.) R.Br.] to nitrogen and moisture conservation practices. M.Sc. Thesis, Rajasthan Agriculture University, Bikaner.

Das, G., \& Gautam, R. C. (2003). Yield and water-use efficiency of pearl millet as influenced by moisture conservation methods under rainfed conditions. Annals of Agricultural Research, 2(1), 78-81.

Deng, X. P., Shan, L., Zhang, H., \& Turner, N. C. (2006). Improving agricultural water use efficiency in arid and semiarid areas of China. Agricultural Water Management, 80(1-3), 23-40.

Derpsch, R., \& Friedrich, T. (2009). Global overview of conservation agriculture adoption. In: Proceedings, Lead Papers, $4^{\text {th }}$ World Congress on Conservation Agriculture, 4-7 February 2009, New Delhi, India, 429-438.
Devasenapathy, P., SenthilKumar, G., \& Shanmugam, P. M. (2009). Energy management in crop production. Indian Journal of Agronomy, 54(1), 8090.

Dhyani, S. K., Newaj, R., \& Sharma, A. R. (2009). Agroforestry: its relation with agronomy, challenges and opportunities. Indian Journal of Agronomy, 54 (3), 259-266.

Edmeades, D. C. (2003). The long-term effects of manures and fertilizers on soil productivity and quality. Nutrient Cycling and Agro-ecosystem, 66, $165-180$.

Filipovic, D., Silvio, K. Zlatko, G. O., Robert Z., \& Djuro, B. (2006). The possibility of fuel saving and reduction of $\mathrm{CO}_{2}$ emission in the soil tillage in Croatia. Agriculture, Ecosystem and Environment, 115, 290-294.

Gathala, M. K., Ladha, J. K., Saharawat, Y. S., Kumar, V., \& Sharma, P. K. (2011a). Effect of tillage and crop establishment methods on physical properties of a medium-textured soil under a seven year rice-wheat rotation. SASAJ, 75, 1851-1862.

Gathala, M. K., Timsina, J., Islam, Md. S., Krupnik, T. J., Bose, T. R., Islam, N., ... McDonald, A. (2016). Productivity, profitability and energetics: a multi-criteria assessment of farmers' tillage and crop establishment options for maize in intensively cultivated environments of South Asia. Field Crops Research, 186, 32-46.

Gathala, M. K., Kumar, V., Sharma, P. C., Saharawat, Y. S., Jat, H. S., Singh, M., ... Ladha, J. K. (2013). Optimizing intense cereal based cropping systems addressing current and future drivers of agricultural change in the north-western Indo-Gangetic Plains of India. Agriculture, Ecosystems \& Environment, 197, 95-97.

Gathala, M. K., Ladha, J. K., Kumar, V., Saharawat, Y. S., Kumar, V., Sharma, P. K., ... Pathak, H. (2011b). Tillage and crop establishment affects sustainability of South Asian rice-wheat system. Agronomy Journal, 103, 961-971.

Gill, M. S., \& Ahlawat, I. P. S. (2006). Crop diversification - its role towards sustainability and profitability. Indian Journal of Fertilizers, 2(9), 125138.

Gomez, K. A., \& Gomez, A. A. (1984). Statistical Procedures for Agricultural Research. John Wiley and Sons, NY.

Grace, P. R., Jain, M. C. Harrington, L., \& Philip, R. (2003). The long-term sustainability of tropical and sub-tropical rice and wheat systems. An environmental perspective (in) improving the productivity and sustainability of rice-wheat systems: issues and impacts. ASA Special Publication, 65, Madison, USA.

Gupta, R. K., Jat, M. L., Gopal, R., \& Kumar, R. (2010). Conservation agriculture based resource management approaches for food and livelihood security. In: Proceedings of the XIX National Symposium on Resource Management Approaches towards Livelihood Security. Indian Society of Agronomy, Division of Agronomy, Indian Agricultural Research Institute, New Delhi.

Hariram, Y. S., Saini, K. S., Kler, D. S, Timsina, J., \& Humphreys, E. (2012). Agronomic and economic evaluation of permanent raised beds, no-tillage and straw mulching for an irrigated maize-wheat system in north-west India. Experimental Agriculture, 48(1), 21-38.

Hobbs, P. R. (2007). Conservation agriculture: what is it and why is it important for future sustainable food production? Journal of Agricultural Science, 145, 127-138.

Jat, R. K., Sapkota, T. B., Singh, R. G. Jat, M. L., Kumar, M., \& Gupta, R. K. (2014). Surveys of conservation agriculture in rice-wheat rotation of Eastern-Gangetic Plains of South Asia. Yield trends and economic profitability. Field Crops Research, 164, 199-210.

Jin, S., \& Yibing, W. (2001). Effect of straw cover on wheat yield and soil environment in dryland field [J]. Transactions of the Chinese Society of Agricultural Engineering, 6.

Jones, R. B., Wendt, J. W., Bunderson, W. T., \& Itimu, O. A. (1996). Leucaena-maize alley cropping in Malawi. Part I: Effects of N, P and leaf application on maize yields and soil properties. Agroforestry Systems, 33 (3), 281-294

Kumar, V., Singh, S., Rajender, S., Chhakar, S., Mallik, R. K., Barainard, D. C., \& Ladha, J. K. (2013a). Weed management strategies to reduce 
herbicide use in zero-till rice-wheat cropping systems of Indo-Gangetic Plains. Weed Technology, 27, 241-254.

Kumar, V., Saharawat, Y. S., Gathala, M. K., Jat, A. S., Singh, S. K., Chaudhary, N., \& Jat, M. L. (2013b). Effect of different tillage and seeding methods on EUE and productivity of wheat in the Indo-Gangetic Plains. Field Crop Research, 142, 1-8.

Lal, M., Bhati, D. S., \& Nag, A. K. (2004). Economics and production potential of different cropping sequence on farmers' field. Journal of EcoPhysiology, 7(3/4), 143-145.

Lehria, S. K., Bali, S. V., \& Singh, B. (2006). Effect of green leaf manure of subabul (Leucaena leucocephala Lam. De Wit.) on maize crop and its residual effect on wheat crop. In: Verma, K.S., Khurana, D.K and Christersson, I. (eds.), Proceedings, IUFROS-ISTS l-UHF International Conference on World Perspective on Short-rotation Forestry for Industrial and Rural Development (pp. 316-319), Nauni, Solan, India.

Mandal, K. G., Saha, K. P., Ghosh, P. K., Hati, K. M., \& Bandyopadhyay, K. K. (2002). Bioenergy and economic analysis of soybean-based crop production systems in central India. Biomass and Bioenergy, 23, 337-345.

McMaster, G. S., Palic, D. B., \& Dunn, G. H. (2002). Soil management alters seedling emergence and subsequent autumn growth and yield in dryland winter wheat-fallow systems in the Central Great Plains on a clay loam soil. Soil and Tillage Research, 65, 193-206.

Michael, A. M. (2014). Water requirement of crops and irrigation management, pp. 478-553. In: Irrigation Theory and Practice, 2nd Edition, Vikas Publishing House, India.

Peoples, M. B., Herridge, D. F., \& Ladha, J. K. (1995). Biological nitrogen fixation: an efficient source of nitrogen for sustainable agriculture production. Plant and Soil, 174, 3-28.

Pingali, M., Vignozzi, N., \& Pellegrini, S. (2004). Soil structure and the effect of management practices. Soil and Tillage Research, 79, 131-143.

Pittelkow, C. M., Liang, X., Linquist, B. A., Groenigen, K. J. V., Lee, J., Lundy, M. E., ... Kessel, C. V. (2014). Productivity limits and potentials of the principles of conservation agriculture. Nature, 517(7534), 365.

Prasad, C., \& Bhatia, P. C. (2009). Farming system research and extension approaches. In: Suraj, B., Karale, R. L., Singh, S., Bharati, K., and Subramaniyan, S. (eds.), Conservation Farming (pp. 429-445). Soil Conservation Society of India, New Delhi.

Prasad, R., Shivay, Y. S., Kumar, D., \& Sharma, S. N. (2006). Learning by doing exercises in soil fertility - A practical manual for soil fertility (pp. 168). Division of Agronomy, Indian Agricultural Research Institute, New Delhi.

Rao, A. S., Singh, R. S., Joshi, N. L., \& Ramakrishna, Y. S. (2000). Evapotranspiration, water and radiation-utilization of cluster bean (Cyamopsis tetragonoloba). Indian Journal of Agricultural Sciences, 70(3), 149-153.

Reddy, T. Y., \& Reddi, G. H. (2009). Agronomy of field crops ( $3^{\text {rd }}$ ed.). Kalyani Publishers. Kalyani Publishers, Ludhiana, India. pp. 9-124.

Reicosky, D. C. (2003). Conservation agriculture: Global environmental benefits of soil carbon management. In Conservation Agriculture (pp. 312). Springer, Dordrecht.

Rockström, J., Karlberg, L., Wani, S. P., Barron, J., Hatibu, N., Oweis, T., Bruggeman, A. J., \& Qiang, Z. (2010). Managing water in rainfed agriculture - The need for a paradigm shift. Agricultural Water Management, 97(4), 543-550.

Saha, S., Chakraborty, D., Sharma, A. R., Tomar, R. K., Bhadraray, S., Sen, U., ... Kalra, N. (2010). Effect of tillage and residue management on soil physical properties and crop productivity in maize (Zea mays)- Indian mustard (Brassica juncea) system. Indian Journal of Agricultural Sciences, 80(8), 679-685.

Sangar, S., Abrol, I. P., \& Gupta. R. K. (2005). Conservation agriculture: conserving resources - enhancing productivity (p. 19). Centre for Advancement of Sustainable Agriculture, National Agriculture Science Complex, New Delhi.

Saharawat, Y. S., Singh, B., Mallik, R. K., Ladha, J. K., Gathala, M. K., Jat, M. L., \& Kumar, V. (2010). Evaluation of alternative tillage and crop establishment methods in a rice-wheat rotation in north-western IndoGangetic Plains. Field Crop Research, 116, 260-267.
Sarker, S. C., Patra, P. S., Mula, G., \& Paramanik, B. (2011). A study on wheat cultivation under zero tillage and conventional tillage practices. Journal of Crop and Weed, 7(1), 33-36.

Saxena, A., Singh, D. V., \& Joshi, N. L. (1997). Effects of tillage and cropping systems on soil moisture balance and pearl millet yield. Journal of Agronomy and Crop Science, 178, 251-257.

Sayre, K. D., \& Hobbs, P. R. (2004). The raised bed systems of cultivation for irrigated production conditions. In: Lal, R., Hobbs, P., Uphoff, N., \& Hansen, D. O. (eds), Sustainable agriculture and the rice-wheat system (pp. 337-355). Ohio State University, Columbus, OH, USA.

Sharma, A. R., Singh, R., Dhyani, S. K., \& Dube, R. K. (2010). Moisture conservation and nitrogen recycling through legume mulching in rainfed maize (Zea mays)-wheat (Triticum aestivum) cropping system. Nutrient Cycling in Agroecosystems, 87(2), 187-197.

Sharma, A. R., Singh, R., Dhyani, S. K., \& Dube, R. K. (2011). Agronomic and economic evaluation of mulching on rainfed-maize-wheat cropping system on the western Himalayan region of India. Journal of Crop Improvement, 25(4), 392-408.

Sharma, N. K., Singh, P. N., Tyagi, P. C., \& Mohan, S. C. (1998). Leucaena mulch on soil-water use and wheat yield. Agricultural Water Management, 35(3), 191-200.

Sharma, P. K., \& Acharya, C. L. (2000). Carry-over of residual soil moisture with mulching and conservation tillage practices for sowing of rainfed wheat (Triticum aestivum) in north-west India. Soil and Tillage Research, 57, 43-52.

Sharma, P. K., Verma, T. S., \& Bhagat, R. M. (1995). Soil structural improvements with the addition of Lantana camera biomass in rice-wheat cropping. Soil Use and Management, 11, 199-203.

Sharma, A. R., \& Behera, U. K. (2009). Nitrogen contribution through Sesbania green manure and dual-purpose legumes in maize-wheat cropping system: agronomic and economic considerations. Plant and Soil, 325(1/2), 289-304.

Sharma, R. K., Chhokar, R. S., Rani, V., Gathais, M. K., \& Kumar, A. (2002). Productivity, economics, energy requirement of rice-wheat system. Proceedings, International Symposium on Herbicide Resistance Management and Zero Tillage in Rice-Wheat Cropping System (pp. 131135). March 4-6. CCS Haryana Agricultural University, Hisar, India.

Sidhu, H. S., Singh, M., Humphreys, E., Singh, Y., Singh, B., Dhilon, S. S., Blackwell, J., Bector, V., Singh, M., \& Singh, S. (2007). The Happy Seeder enables direct drilling of wheat into rice stubble. Australian Journal of Experimental Agriculture, 47, 844-854.

Singh, D., \& Singh, R. (1995). Effect of levels and placement of phosphorus on moisture use in pigeonpea-based intercropping system under dryland conditions. Annals of Agricultural Research 16(3), 289-291.

Singh, R., Singh, B., \& Patidar, M. (2008). Effect of preceding crops and nutrient management on productivity of wheat (Triticum aestivum)-based cropping system in arid region. Indian Journal of Agronomy, 53(4), 267272.

Singh, V., Sharma, S. K., Deo, R., Siag, R. K., \& Verma, B. L. (1998). Performance of different crop sequences under various irrigation levels. Indian Journal of Agronomy, 35, 287-296.

Singh, Y., Singh, B., \& Timsina, J. (2005). Crop residue management for nutrient cycling and improving soil productivity in rice-based cropping systems in the tropics. Advances in Agronomy, 85, 269-407.

Solanki, N. S., \& Sahu, M. P. (2007). Productivity and P-use efficiency of cluster bean (Cyamopsis tetragonoloba) as influenced by bio-regulators and phosphorus. Indian Journal of Agronomy, 52(3), 143-147.

Timsina, J., Jat, M. L., \& Majumdar, K. (2010). Rice-maize systems of South Asia: current status, future prospects and research priorities for nutrient management. Plant Soil, 325, 65-82.

Verhulst, N., Deckers, J., \& Govaerts, B. (2009). Classification of the soil at CIMMYT's experimental station in the Yaqui Valley near Ciudad Obregón, Sonora, Mexico. CIMMYT Report. CIMMYT, Mexico, pp. 10.

Vyas, S. H., Patel, J. C, Patel, B. S., \& Khanpara, V. S. (1994). Influence of irrigation and $\mathrm{N}$ and $\mathrm{P}$ fertilization on yield, consumptive use of water, water use efficiency and nutrient uptake by rainy-season pearl millet in 
south Saurashtra region. Gujarat Agricultural University Research Journal, 19(2), 113-116.

Wanga, X., Daia, K., Wanga, Y., Zhanga, X., Zhaoa, Q., Wua, X., ... Oenemad, O. (2010). Nutrient management adaptation for dryland maize yields and water use efficiency to long-term rainfall variability in China. Agricultural Water Management, 97, 1344-1350.

Yadav, P. K., Kumar, S., \& Kumar, M. (2009). Sustaining yield of pearl milletwheat cropping system in semi-arid conditions. Haryana Journal of Agronomy, 25(1/2), 1-3. 
Table S1. Economics and input/output energy $\left(\mathrm{MJ} \mathrm{ha}^{-1}\right)$ for different crops and cropping systems (mean values for 2010-2011 and 2011-2012).

\begin{tabular}{|c|c|c|c|c|c|c|c|c|}
\hline \multirow{2}{*}{\multicolumn{2}{|c|}{$\begin{array}{l}\text { Particulars } \\
\text { A. Inputs (fixed cost) }\end{array}$}} & \multicolumn{2}{|l|}{ Pearl millet } & \multicolumn{2}{|l|}{ Cluster bean } & \multicolumn{2}{|l|}{ Green gram } & \multirow{2}{*}{$\begin{array}{l}* * \text { Energy } \\
\text { coefficients }\end{array}$} \\
\hline & & $\begin{array}{l}\text { Unit } \\
\text { expenditure }\end{array}$ & $\begin{array}{l}\text { Energy } \\
(\mathbf{M J})\end{array}$ & $\begin{array}{l}\text { Unit } \\
\text { expenditure }\end{array}$ & $\begin{array}{l}\text { Energy } \\
\text { (MJ) }\end{array}$ & $\begin{array}{l}\text { Unit } \\
\text { expenditure }\end{array}$ & $\begin{array}{l}\text { Energy } \\
\text { (МJ) }\end{array}$ & \\
\hline 1 & Seed & $4 \mathrm{~kg}$ & 58.8 & $30 \mathrm{~kg}$ & 441.0 & $40 \mathrm{~kg}$ & 588.0 & $14.7 \mathrm{MJ} / \mathrm{kg}$ \\
\hline 2 & $\begin{array}{l}\text { Sowing (Happy- } \\
\text { seeder) }\end{array}$ & $3 \mathrm{hrs} / \mathrm{ha}$ & 675.7 & $3 \mathrm{hrs} / \mathrm{ha}$ & 675.7 & $3 \mathrm{hrs} / \mathrm{ha}$ & 675.7 & $4 \mathrm{l} / \mathrm{hr}(56.3 \mathrm{MJ} / \mathrm{l})$ \\
\hline \multirow[t]{4}{*}{3} & Fertilizer & & & & & & & \\
\hline & i. $\mathrm{N}$ & $60 \mathrm{~kg}$ & 3636.0 & $20 \mathrm{~kg}$ & 1212.0 & $20 \mathrm{~kg}$ & 1212.0 & $60.6 \mathrm{MJ} / \mathrm{kg}$ \\
\hline & ii. $\mathrm{P}$ & $40 \mathrm{~kg}$ & 444.0 & $40 \mathrm{~kg}$ & 444.0 & $40 \mathrm{~kg}$ & 444.0 & $11.1 \mathrm{MJ} / \mathrm{kg}$ \\
\hline & iii. $\mathrm{K}$ & $20 \mathrm{~kg}$ & 134.0 & $20 \mathrm{~kg}$ & 134.0 & $20 \mathrm{~kg}$ & 134.0 & $6.7 \mathrm{MJ} / \mathrm{kg}$ \\
\hline \multirow[t]{4}{*}{4} & $\begin{array}{l}\text { Herbicides and } \\
\text { application }\end{array}$ & & & & & & & \\
\hline & I. Pre-sowing & 0.751 & 135.0 & 0.751 & 135.0 & 0.751 & 135.0 & $120 \mathrm{MJ} / \mathrm{kg}$ \\
\hline & ii. After sowing & 1.51 & 180.0 & 1.51 & 180.0 & 1.51 & 180.0 & $120 \mathrm{MJ} / \mathrm{kg} \mathrm{SC} *$ \\
\hline & iii. Application & 2 laborers & 31.4 & 2 laborers & 31.4 & 2 laborers & 31.4 & $1.96 \mathrm{MJ} / \mathrm{man}-\mathrm{hr}$ \\
\hline 5 & $\begin{array}{l}\text { Gap filling and } \\
\text { thinning }\end{array}$ & 3 laborers & 47.0 & 2 laborers & 31.4 & 2 laborers & 31.4 & $1.96 \mathrm{MJ} / \mathrm{man}-\mathrm{hr}$ \\
\hline 6 & Hand weeding & 0 & 0.0 & 5 laborers & 78.4 & 0 & 0.0 & $1.96 \mathrm{MJ} / \mathrm{man}-\mathrm{hr}$ \\
\hline \multirow[t]{2}{*}{7} & i. Insecticide & 0 & 0.0 & 21 & 240.0 & 21 & 240.0 & $120 \mathrm{MJ} / \mathrm{kg} \mathrm{SC} *$ \\
\hline & iii. Application & 0 & 0.0 & 2 laborers & 31.4 & 2 laborers & 31.4 & $1.96 \mathrm{MJ} / \mathrm{man}-\mathrm{hr}$ \\
\hline 8 & $\begin{array}{l}\text { Bird watching (15 } \\
\text { days) }\end{array}$ & 15 laborers & 235.2 & 0 & 0.0 & 0 & 0.0 & $1.96 \mathrm{MJ} / \mathrm{man}-\mathrm{hr}$ \\
\hline 9 & Harvesting & 10 laborers & 156.8 & 10 laborers & 156.8 & 10 laborers & 156.8 & $1.96 \mathrm{MJ} / \mathrm{man}-\mathrm{hr}$ \\
\hline \multirow[t]{2}{*}{10} & Threshing & 5 laborers & 78.4 & 0 & 0.0 & 3 laborers & 47.0 & $1.96 \mathrm{MJ} / \mathrm{man}-\mathrm{hr}$ \\
\hline & Total & & 5812.3 & & 3791.0 & & 3906.6 & \\
\hline \multicolumn{9}{|c|}{ B. Inputs (Variable cost) } \\
\hline \multicolumn{9}{|c|}{1 Crop residues } \\
\hline & i. Amount & $5.0 \mathrm{t} / \mathrm{ha}$ dry mass & 62500.0 & $5.0 \mathrm{t} / \mathrm{ha}$ dry mass & 62500.0 & $5.0 \mathrm{t} / \mathrm{ha}$ dry mass & 62500.0 & $12.5 \mathrm{MJ} / \mathrm{kg}$ \\
\hline & ii. Application cost & 3 laborers & 47.0 & 3 laborers & 47.0 & 3 laborers & 47.0 & $1.96 \mathrm{MJ} / \mathrm{man}-\mathrm{hr}$ \\
\hline & Total & & 62547.0 & & 62547.0 & & 62547.0 & \\
\hline \multirow[t]{4}{*}{2} & Leucaena twigs & & & & & & & \\
\hline & i. Amount & $3.5 \mathrm{t} / \mathrm{ha}$ dry mass & 43750.0 & $3.5 \mathrm{t} / \mathrm{ha}$ dry mass & 43750.0 & $3.5 \mathrm{t} / \mathrm{ha}$ dry mass & 43750.0 & $12.5 \mathrm{MJ} / \mathrm{kg}$ \\
\hline & ii. Application cost & 10 laborers & 156.8 & 10 laborers & 156.8 & 10 laborers & 156.8 & $1.96 \mathrm{MJ} / \mathrm{man}-\mathrm{hr}$ \\
\hline & Total & & 43906.8 & & 43906.8 & & 43906.8 & \\
\hline \multicolumn{9}{|c|}{ C. Output } \\
\hline 1 & Main product & & & & & & & $14.7 \mathrm{MJ} / \mathrm{kg}$ \\
\hline 2 & By-product & & & & & & & $\mathrm{MJ} / \mathrm{kg}$ \\
\hline
\end{tabular}

SC* $=$ Super chemicals; $* 8$ hours/day

**Energy co-efficient - Source: Devasenapathy et al. (2009) 
Table S2. Economics and input/output energy $\left(\mathrm{MJ} \mathrm{ha}^{-1}\right)$ of winter season crops (mean values for 2010-2011 and 2011-2012).

\begin{tabular}{|c|c|c|c|c|c|c|c|c|}
\hline \multirow{2}{*}{\multicolumn{2}{|c|}{$\begin{array}{l}\text { Particulars } \\
\text { A. Inputs (fixed cost) }\end{array}$}} & \multicolumn{2}{|l|}{ Wheat } & \multicolumn{2}{|l|}{ Chickpea } & \multicolumn{2}{|l|}{ Mustard } & \multirow{2}{*}{$\begin{array}{l}\text { Energy } \\
\text { coefficients }\end{array}$} \\
\hline & & $\begin{array}{l}\text { Unit } \\
\text { expenditure }\end{array}$ & $\begin{array}{l}\text { Energy } \\
\text { (MJ) }\end{array}$ & $\begin{array}{l}\text { Unit } \\
\text { expenditure }\end{array}$ & $\begin{array}{l}\text { Energy } \\
\text { (MJ) }\end{array}$ & $\begin{array}{l}\text { Unit } \\
\text { expenditure }\end{array}$ & $\begin{array}{l}\text { Energy } \\
\text { (MJ) }\end{array}$ & \\
\hline 1 & Seed & $120 \mathrm{~kg}$ & 1764.0 & $80 \mathrm{~kg}$ & 1176.0 & $4 \mathrm{~kg}$ & 100.0 & $14.7 \mathrm{MJ} / \mathrm{kg}$ \\
\hline 2 & Sowing (Happy-seeder) & $3 \mathrm{hrs} / \mathrm{ha}$ & 675.7 & $3 \mathrm{hrs} / \mathrm{ha}$ & 675.7 & $3 \mathrm{hrs} / \mathrm{ha}$ & 675.7 & $41 / \mathrm{hr}(56.3 \mathrm{MJ} / 1)$ \\
\hline \multirow[t]{4}{*}{3} & Fertilizer & & & & & & & \\
\hline & i. $\mathrm{N}$ & $60 \mathrm{~kg}$ & 3636.0 & $20 \mathrm{~kg}$ & 1212.0 & $60 \mathrm{~kg}$ & 3636.0 & $60.6 \mathrm{MJ} / \mathrm{kg}$ \\
\hline & ii. $P$ & $40 \mathrm{~kg}$ & 444.0 & $40 \mathrm{~kg}$ & 444.0 & $40 \mathrm{~kg}$ & 444.0 & $11.1 \mathrm{MJ} / \mathrm{kg}$ \\
\hline & iii. $\mathrm{K}$ & $20 \mathrm{~kg}$ & 134.0 & $20 \mathrm{~kg}$ & 134.0 & $20 \mathrm{~kg}$ & 134.0 & $6.7 \mathrm{MJ} / \mathrm{kg}$ \\
\hline \multirow[t]{4}{*}{4} & Herbicides and application & & & & & & & \\
\hline & I. Pre-sowing & 1.51 & 180.0 & 1.51 & 180.0 & 1.51 & 180.0 & $120 \mathrm{MJ} / \mathrm{kg} \mathrm{SC} *$ \\
\hline & II. Post-emergence & 1.51 & 180.0 & 0 & 0.0 & 0 & 0.0 & $120 \mathrm{MJ} / \mathrm{kg} \mathrm{SC} *$ \\
\hline & iii. Application ( 8 hrs/day) & 2 laborers* & 31.4 & 1 laborer & 15.7 & 1 laborer & 15.7 & $1.96 \mathrm{MJ} / \mathrm{man}-\mathrm{hr}$ \\
\hline 5 & Gap filling and thinning & 0 & 0.0 & 3 laborers & 47.0 & 3 laborers & 47.0 & $1.96 \mathrm{MJ} / \mathrm{man}-\mathrm{hr}$ \\
\hline \multirow[t]{2}{*}{6} & i. Insecticide & 0 & 0.0 & 11 & 120.0 & 11 & 120.0 & $120 \mathrm{MJ} / \mathrm{kg} \mathrm{SC} *$ \\
\hline & ii. Application & 0 & 0.0 & 1 laborer & 15.7 & 1 laborer & 15.7 & $1.96 \mathrm{MJ} / \mathrm{man}-\mathrm{hr}$ \\
\hline \multirow[t]{2}{*}{7} & i. Irrigation amount & 1 irrigation & 3.0 & 1 row irrigation & 3.0 & 1 row irrigation & 3.0 & $\begin{array}{l}11.93 \mathrm{MJ} / \mathrm{KWh}( \\
0.37 \mathrm{MJ} / \mathrm{hr})\end{array}$ \\
\hline & ii. Application & 1 laborer & 15.7 & 1 laborer & 15.7 & 1 laborer & 15.7 & $1.96 \mathrm{MJ} / \mathrm{man}-\mathrm{hr}$ \\
\hline 8 & Bird watching (15 days) & 15 laborers & 235.2 & 15 laborers & 235.2 & 15 laborers & 235.2 & $1.96 \mathrm{MJ} / \mathrm{man}-\mathrm{hr}$ \\
\hline 9 & Harvesting & 10 laborers & 156.8 & 8 laborers & 125.6 & 10 laborers & 156.8 & $1.96 \mathrm{MJ} / \mathrm{man}-\mathrm{hr}$ \\
\hline \multirow[t]{2}{*}{10} & Threshing & 5 laborers & 78.4 & 3 laborers & 47.0 & 5 laborers & 78.4 & $1.96 \mathrm{MJ} / \mathrm{man}-\mathrm{hr}$ \\
\hline & Total & & 7534.2 & & 4446.6 & & 5857.2 & \\
\hline \multicolumn{9}{|c|}{ B. Inputs (Variable cost) } \\
\hline \multirow{4}{*}{1} & Crop residues & & & & & & & \\
\hline & i. Amount & $\begin{array}{l}5.0 \mathrm{t} / \mathrm{ha} \text { dry } \\
\text { mass }\end{array}$ & 62500.0 & $\begin{array}{l}5.0 \mathrm{t} / \text { ha dry } \\
\text { mass }\end{array}$ & 62500.0 & $\begin{array}{l}5.0 \mathrm{t} / \mathrm{ha} \text { dry } \\
\text { mass }\end{array}$ & 62500.0 & $12.5 \mathrm{MJ} / \mathrm{kg}$ \\
\hline & ii. Application & 3 laborers & 47.0 & 3 laborers & 47.0 & 3 laborers & 47.0 & $1.96 \mathrm{MJ} / \mathrm{man}-\mathrm{hr}$ \\
\hline & Total & & 62547.0 & & 62547.0 & & 62547.0 & \\
\hline \multirow[t]{4}{*}{2} & Leucaena twigs & & & & & & & \\
\hline & i. Amount (dry biomass) & $3.5 \mathrm{t} / \mathrm{ha}$ & 43750.0 & $3.5 \mathrm{t} / \mathrm{ha}$ & 43750.0 & $3.5 \mathrm{t} / \mathrm{ha}$ & 43750.0 & $12.5 \mathrm{MJ} / \mathrm{kg}$ \\
\hline & ii. Application & 10 laborers & 156.8 & 10 laborers & 156.8 & 10 laborers & 156.8 & $1.96 \mathrm{MJ} / \mathrm{man}-\mathrm{hr}$ \\
\hline & Total & & 43906.8 & & 43906.8 & & 43906.8 & \\
\hline \multicolumn{9}{|c|}{ C. Output } \\
\hline 1 & $\begin{array}{l}\text { Main product (all crops except } \\
\text { oilseeds) }\end{array}$ & & & & & & \multicolumn{2}{|c|}{$14.7 \mathrm{MJ} / \mathrm{kg}(25 \mathrm{MJ} / \mathrm{kg}$ in oilseeds $)$} \\
\hline 2 & By-product (all crops) & & & & & & $12.5 \mathrm{MJ} / \mathrm{k}$ & \\
\hline
\end{tabular}

